\title{
ON THE QUOTIENT SHAPES OF VECTORIAL SPACES
}

\author{
NIKICA UGLEŠIĆ
}

To my Master teacher Sibe Mardešić - with homage and gratitude

\begin{abstract}
The quotient shapes of vectorial spaces are considered - algebraically and topologically, especially, of the normed spaces. In the algebraic case, all the shape classifications and the isomorphism classification coincide. However, in the general topological case and, especially, in the normed case, the quotient shape classifications are strictly coarser than the isomorphism classification.
\end{abstract}

\section{IntRoduction}

The shape theory (for compacta in the Hilbert cube) was founded by K. Borsuk, [1]. The theory was rapidly developed and generalized by many authors. The main references are [2], [3], [5] and, especially, [9]. Although, in general, founded purely categorically, a shape theory is mostly well known only as the (standard) shape theory of topological spaces with respect to spaces having homotopy types of polyhedra. The generalizations founded in [8] and [11] are, primarily, also on that line.

The quotient shape theory was recently introduced by the author, [10]. It is, of course, a kind of the general (abstract) shape theory, [9], I. 2. However, it is possible and non-trivial, and can be straightforwardly developed for every concrete category $\mathcal{C}$ and for every infinite cardinal $\kappa \geq \aleph_{0}$. Concerning a shape of objects, in general, one has to decide which ones are "nice" absolutely and/or relatively (with respect to a chosen one). In this approach, the main principle reads as follows: An object is "nice" if it is isomorphic to a quotient object belonging to a special full subcategory and if it (its "basis") has cardinality less than (less than or equal to) a given infinite cardinal. It leads to the basic idea: to approximate a $\mathcal{C}$-object $X$ by a suitable inverse system consisting of its quotient objects $X_{\lambda}$ (and the quotient morphisms)

2010 Mathematics Subject Classification. Primary 54E99; Secondary 55P55.

Key words and phrases. Concrete category, (quotient) shape, (topological, normed) vectorial space, (continuous) linear function, quotient (topological, normed) vectorial space, dimension, infinite cardinal. 
which have cardinalities less than (less or equal to) $\kappa$. Such an approximation exists in the form of any $\kappa^{-}$-expansion ( $\kappa$-expansion) of $X$,

$$
\begin{gathered}
\boldsymbol{p}_{\kappa^{-}}=\left(p_{\lambda}\right): X \rightarrow \boldsymbol{X}_{\kappa^{-}}=\left(X_{\lambda}, p_{\lambda \lambda^{\prime}}, \Lambda_{\kappa^{-}}\right) \\
\left(\boldsymbol{p}_{\kappa}=\left(p_{\lambda}\right): X \rightarrow \boldsymbol{X}_{\kappa}=\left(X_{\lambda}, p_{\lambda \lambda^{\prime}}, \Lambda_{\kappa}\right)\right),
\end{gathered}
$$

where $\boldsymbol{X}_{\kappa^{-}}\left(\boldsymbol{X}_{\kappa}\right)$ belongs to the subcategory pro- $\mathcal{D}_{\kappa^{-}}\left(\right.$pro- $\left.\mathcal{D}_{\kappa}\right)$ of pro- $\mathcal{D}$, and $\mathcal{D}_{\kappa^{-}}\left(\mathcal{D}_{\kappa}\right)$ is the subcategory of $\mathcal{D}$ determined by all the objects having cardinalities less than (less or equal to) $\kappa$, while $\mathcal{D}$ is a full subcategory of $\mathcal{C}$. Clearly, if $X \in O b \mathcal{D}$ and the cardinality $|X|<\kappa(|X| \leq \kappa)$, then the rudimentary pro-morphism $\left\lfloor 1_{X}\right\rfloor: X \rightarrow\lfloor X\rfloor$ is a $\kappa^{-}$-expansion ( $\kappa$-expansion) of $X$. The corresponding shape category $S h_{\mathcal{D}_{\kappa^{-}}}(\mathcal{C})\left(S h_{\mathcal{D}_{\kappa}}(\mathcal{C})\right)$ and shape functor $S_{\kappa^{-}}: \mathcal{C} \rightarrow S h_{\mathcal{D}_{\kappa^{-}}}(\mathcal{C})\left(S_{\kappa}: \mathcal{C} \rightarrow S h_{\mathcal{D}_{\kappa}}(\mathcal{C})\right)$ exist by the general (abstract) shape theory, and they have all the appropriate general properties. Moreover, there exist the relating functors $S_{\kappa^{-} \kappa}: S h_{\mathcal{D}_{\kappa}}(\mathcal{C}) \rightarrow S h_{\mathcal{D}_{\kappa^{-}}}(\mathcal{C})$ and $S_{\kappa \kappa^{\prime}}: S h_{\mathcal{D}_{\kappa^{\prime}}}(\mathcal{C}) \rightarrow S h_{\mathcal{D}_{\kappa}}(\mathcal{C}), \kappa \leq \kappa^{\prime}$, such that $S_{\kappa^{-} \kappa^{\prime}} S_{\kappa}=S_{\kappa^{-}}$and $S_{\kappa \kappa^{\prime}} S_{\kappa^{\prime}}=$ $S_{\kappa}$. We should mention that the simplest and very often interesting case is even $\mathcal{D}=\mathcal{C}$. In such a case we simplify the notation $S h_{\mathcal{D}_{\kappa^{-}}}(\mathcal{C})\left(S h_{\mathcal{D}_{\kappa}}(\mathcal{C})\right)$ to $S h_{\kappa^{-}}(\mathcal{C})\left(S h_{\kappa}(\mathcal{C})\right)$ or to $S h_{\kappa^{-}}\left(S h_{\kappa}\right)$ when $\mathcal{C}$ is fixed.

In [10], several well known concrete categories were considered and many examples are given which show that the quotient shape theory yields classifications strictly coarser than those by isomorphisms. In this paper we continue the consideration of quotient shapes of vectorial spaces ([10], Section 12), especially, of the topological and normed vectorial spaces.

\section{Preliminaries}

We shall not repeat the construction of an abstract shape category given in [9]. Further, our category theory language follows [6], while all necessary facts concerning ordinals and cardinals one can find in [4], Chapter II. Nevertheless, we have to recall some indispensable notions and constructions which are introduced or exhibited in [10].

Given a category pair $(\mathcal{C}, \mathcal{D})$, where $\mathcal{D} \subseteq \mathcal{C}$ is full, and a cardinal $\kappa$, let $\mathcal{D}_{\kappa^{-}}$ $\left(\mathcal{D}_{\kappa}\right)$ denote the full subcategory of $\mathcal{D}$ determined by all the objects having cardinalities or, in some special cases, the cardinalities of "bases" less than (less or equal to) $\kappa$. By following the main principle, let $\left(\mathcal{C}, \mathcal{D}_{\kappa^{-}}\right)\left(\left(\mathcal{C}, \mathcal{D}_{\kappa}\right)\right)$ be such a pair of concrete categories. If

(a) every $\mathcal{C}$-object $(X, \sigma)$ admits a directed set $R\left(X, \sigma, \kappa^{-}\right) \equiv \Lambda_{\kappa^{-}}$ $\left(R(X, \sigma, \kappa) \equiv \Lambda_{\kappa}\right)$ of equivalence relations $\lambda$ on $X$ such that each quotient object $\left(X / \lambda, \sigma_{\lambda}\right)$ has to belong to $\mathcal{D}_{\kappa^{-}}\left(\mathcal{D}_{\kappa}\right)$, while each quotient morphism $p_{\lambda}:(X, \sigma) \rightarrow\left(X / \lambda, \sigma_{\lambda}\right)$ has to belong to $\mathcal{C}$;

(b) the induced morphisms between quotient objects belong to $\mathcal{D}_{\kappa^{-}}\left(\mathcal{D}_{\kappa}\right)$; 
(c) every morphism $f:(X, \sigma) \rightarrow(Y, \tau)$ of $\mathcal{C}$, having the codomain in $\mathcal{D}_{\kappa^{-}}$ $\left(\mathcal{D}_{\kappa}\right)$, factorizes uniquely through a quotient $p_{\lambda}:(X, \sigma) \rightarrow\left(X / \lambda, \sigma_{\lambda}\right)$, $f=g p_{\lambda}$, with $g$ belonging to $\mathcal{D}_{\kappa^{-}}\left(\mathcal{D}_{\kappa}\right)$,

then $\mathcal{D}_{\kappa^{-}}\left(\mathcal{D}_{\kappa}\right)$ is a pro-reflective subcategory of $\mathcal{C}$. Consequently, there exists a (nontrivial) "quotient shape" category $S h_{\left(\mathcal{C}, \mathcal{D}_{\kappa^{-}}\right)} \equiv S h_{\mathcal{D}_{\kappa^{-}}}(\mathcal{C})\left(S h_{\left(\mathcal{C}, \mathcal{D}_{\kappa}\right)} \equiv\right.$ $\left.S h_{\mathcal{D}_{\kappa}}(\mathcal{C})\right)$ obtained by the general construction.

Therefore, a $\kappa^{-}$-shape morphism $F_{\kappa^{-}}:(X, \sigma) \rightarrow(Y, \tau)$ is represented by a diagram (in pro- $\mathcal{C}$ )

$$
\begin{array}{ccc}
(\boldsymbol{X}, \boldsymbol{\sigma})_{\kappa^{-}} & p_{\mathcal{\kappa}^{-}} & (X, \sigma) \\
\boldsymbol{f}_{\kappa^{-}} \downarrow & & \\
(\boldsymbol{Y}, \boldsymbol{\tau})_{\kappa^{-}} & \stackrel{q_{\kappa^{-}}}{\leftarrow} & (Y, \tau)
\end{array}
$$

(with $\boldsymbol{p}_{\kappa^{-}}$and $\boldsymbol{q}_{\kappa^{-}}$- a pair of appropriate expansions), and similarly for a $\kappa$-shape morphism $F_{\kappa}:(X, \sigma) \rightarrow(Y, \tau)$. Since all $\mathcal{D}_{\kappa^{-}}$-expansions $\left(\mathcal{D}_{\kappa^{-}}\right.$ expansions) of a $\mathcal{C}$-object are mutually isomorphic objects of pro- $\mathcal{D}_{\kappa^{-}}$(pro$\mathcal{D}_{\kappa}$ ), the composition and identities follow straightforwardly. Observe that every quotient morphism $p_{\lambda}$ is an effective epimorphism (If $U$ is the forgetful functor, then $U\left(p_{\lambda}\right)$ is a surjection), and thus condition (E2) for an expansion follows trivially.

The corresponding "quotient shape" functors $S_{\kappa^{-}}: \mathcal{C} \rightarrow S h_{\mathcal{D}_{\kappa^{-}}}(\mathcal{C})$ and $S_{\kappa}: \mathcal{C} \rightarrow S h_{\mathcal{D}_{\kappa}}(\mathcal{C})$ are defined in the same general manner. That means,

$$
S_{\kappa^{-}}(X, \sigma)=S_{\kappa}(X, \sigma)=(X, \sigma) ;
$$

if $f:(X, \sigma) \rightarrow(Y, \tau)$ is a $\mathcal{C}$-morphism, then, for every $\mu \in M_{\kappa^{-}}$, the composite $g_{\mu} f:(Y, \tau) \rightarrow\left(Y_{\mu}, \tau_{\mu}\right)$ factorizes (uniquely) through a $p_{\lambda(\mu)}:(X, \sigma) \rightarrow\left(X_{\lambda(\mu)}, \sigma_{\lambda(\mu)}\right)$, and thus, the correspondence $\mu \mapsto \lambda(\mu)$ yields a function $\varphi: M_{\kappa^{-}} \rightarrow \Lambda_{\kappa^{-}}$and a family of $\mathcal{D}_{\kappa^{-}}$-morphisms $f_{\mu}$ : $\left(X_{\varphi(\mu)}, \sigma_{\varphi(\mu)}\right) \rightarrow\left(Y_{\mu}, \tau_{\mu}\right)$ such that $q_{\mu} f=f_{\mu} p_{\varphi(\mu)}$; one easily shows that $\left(\varphi, f_{\mu}\right):(\boldsymbol{X}, \boldsymbol{\sigma})_{\kappa^{-}} \rightarrow(\boldsymbol{Y}, \boldsymbol{\tau})_{\kappa^{-}}$is a morphism of $i n v-\mathcal{D}_{\kappa^{-}}$, so the equivalence class $\boldsymbol{f}_{\kappa^{-}}=\left[\left(\varphi, f_{\mu}\right)\right]:(\boldsymbol{X}, \boldsymbol{\sigma})_{\kappa^{-}} \rightarrow(\boldsymbol{Y}, \boldsymbol{\tau})_{\kappa^{-}}$is a morphism of pro- $\mathcal{D}_{\kappa^{-}}$; then we put $S_{\kappa^{-}}(f)=\left\langle\boldsymbol{f}_{\kappa^{-}}\right\rangle \equiv F_{\kappa^{-}}:(X, \sigma) \rightarrow(Y, \tau)$ in $S h_{\mathcal{D}_{\kappa^{-}}}(\mathcal{C})$. The identities and composition are obviously preserved. In the same way one defines the functor $S_{\kappa}$.

Furthermore, since $(\boldsymbol{X}, \boldsymbol{\sigma})_{\kappa^{-}}$is a subsystem of $(\boldsymbol{X}, \boldsymbol{\sigma})_{\kappa}$, one easily shows that there exists a functor $S_{\kappa^{-} \kappa}: S h_{\mathcal{D}_{\kappa}}(\mathcal{C}) \rightarrow S h_{\mathcal{D}_{\kappa^{-}}}(\mathcal{C})$ such that $S_{\kappa^{-}}{ }_{\kappa} S_{\kappa}=$ $S_{\kappa^{-}}$, i.e., the diagram

$$
S h_{\mathcal{D}_{\kappa^{-}}}(\mathcal{C}) \stackrel{\mathcal{C}}{\swarrow S_{\kappa^{-}}} \underbrace{}_{S_{\kappa^{-} \kappa}} S_{\kappa} \searrow S h_{\mathcal{D}_{\kappa}}(\mathcal{C})
$$

commutes. Moreover, an analogous functor $S_{\kappa \kappa^{\prime}}: S h_{\mathcal{D}_{\kappa^{\prime}}}(\mathcal{C}) \rightarrow S h_{\mathcal{D}_{\kappa}}(\mathcal{C})$, satisfying $S_{\kappa \kappa^{\prime}} S_{\kappa^{\prime}}=S_{\kappa}$, exists for every pair of infinite cardinals $\kappa \leq \kappa^{\prime}$. 
Generally, in the case of $\kappa=\aleph_{0}$, the $\kappa^{-}$-shape is said to be the finite (quotient) shape, because all the objects in the expansions are of finite (bases) cardinalities, and the category is denoted by $S h_{\mathcal{D}_{\underline{0}}}(\mathcal{C})$ or by $S h_{\underline{0}}(\mathcal{C}) \equiv S h_{\underline{0}}$ only, whenever $\mathcal{D}=\mathcal{C}$.

We shall now apply this theory to each of the category $\mathcal{C} \in\left\{\right.$ Vect $_{F}$, $\left.N V e c t_{F}, T V e c t_{F}\right\}$ (with $\mathcal{D}=\mathcal{C}$ ) of vectorial spaces and appropriate linear functions, where $N$ and $T$ suggest a norm and a topology respectively.

\section{The Algebraic Shapes of Vectorial SPACES}

Given a field $F$, let $V e c t_{F}$ denote the category of all vectorial spaces over $F$ and all their linear functions. According to [10], Section 12, we propose that every finite-dimensional vectorial space $X$ is "nice", and if $X$ is infinite- $\kappa$ dimensional, then every vectorial space (over the same field) having dimension less than $\kappa$ is "nice comparing to" $X$. As usually, $\operatorname{dim} X=|B|$, where $B$ is an algebraic (Hamel) basis of $X$. For the sake of completeness, let us briefly recall the canonical construction and the main result of [10], Section 12.

Let $X \in O b\left(V e c t_{F}\right)$ and let $\left\{Z_{\lambda} \mid \lambda \in \Lambda\right\}$ be the set of all its subspaces $Z_{\lambda} \unlhd X$. We define

$$
\lambda \leq \lambda^{\prime} \Leftrightarrow Z_{\lambda^{\prime}} \unlhd Z_{\lambda} .
$$

Then $(\Lambda, \leq)$ is a poset, that is directed because of

$$
Z_{\lambda^{\prime \prime}} \unlhd Z_{\lambda} \cap Z_{\lambda^{\prime}} \Leftrightarrow \lambda^{\prime \prime} \geq \lambda, \lambda^{\prime} .
$$

For every $\lambda \in \Lambda$, put

$$
X_{\lambda} \equiv X / Z_{\lambda}=\left\{[x]_{\lambda}=x+Z_{\lambda} \mid x \in X\right\}
$$

to be the quotient vectorial space, and let

$$
p_{\lambda}: X \rightarrow X_{\lambda}, \quad p_{\lambda}(x)=[x]_{\lambda},
$$

be the canonical projection, which is a linear function. Notice that $\operatorname{dim} X=$ $\operatorname{dim} X_{\lambda}+\operatorname{dim} Z_{\lambda}$. Given a pair $\lambda \leq \lambda^{\prime}$ (i.e., $Z_{\lambda^{\prime}} \unlhd Z_{\lambda}$ ), let

$$
p_{\lambda \lambda^{\prime}}: X_{\lambda^{\prime}} \rightarrow X_{\lambda}, \quad p\left([x]_{\lambda^{\prime}}\right)=[x]_{\lambda},
$$

be the relating function, which is linear as well. Then, $p_{\lambda \lambda^{\prime}} p_{\lambda^{\prime}}=p_{\lambda}$ and $p_{\lambda \lambda^{\prime}} p_{\lambda^{\prime} \lambda^{\prime \prime}}=p_{\lambda \lambda^{\prime \prime}}$, whenever $\lambda \leq \lambda \leq \lambda^{\prime \prime}$. In this way a certain inverse system $\boldsymbol{X}=\left(X_{\lambda}, p_{\lambda \lambda^{\prime}}, \Lambda\right)$ in $V e c t_{F}$ is associated with $X$, as well as, a morphism

$$
\boldsymbol{p}=\left(p_{\lambda}\right): X \rightarrow \boldsymbol{X}
$$

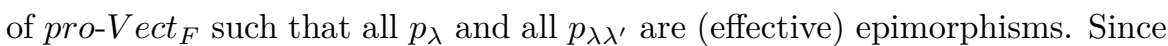
there exists $\lambda_{*}=\max (\Lambda, \leq)=\varnothing$ (corresponding to the trivial subspace $Z_{\lambda_{*}}=\{\theta\}$ ), and $X_{\lambda_{*}} \cong X$, the system $\boldsymbol{X}$ is isomorphic (in pro-Vect pro ) to the rudimentary system $\lfloor X\rfloor$ of $X$. However, given an infinite cardinal $\kappa$, the restriction to the subset $\Lambda_{\kappa^{-}} \subseteq \Lambda\left(\Lambda_{\kappa} \subseteq \Lambda\right)$ of all $\lambda \in \Lambda$ such that $\operatorname{dim} X_{\lambda}<\kappa$ 
( $\left.\operatorname{dim} X_{\lambda} \leq \kappa\right)$, which is directed (it follows, in every detail, by Lemma 3.8 (iii) below; see also the proof of Theorem 4.2 below) as well, yields a morphism

$$
\begin{gathered}
\boldsymbol{p}_{\kappa^{-}}=\left(p_{\lambda}\right): X \rightarrow \boldsymbol{X}_{\kappa^{-}}=\left(X_{\lambda}, p_{\lambda \lambda^{\prime}}, \Lambda_{\kappa^{-}}\right. \\
\left(\boldsymbol{p}_{\kappa}=\left(p_{\lambda}\right): X \rightarrow \boldsymbol{X}_{\kappa}=\left(X_{\lambda}, p_{\lambda \lambda^{\prime}}, \Lambda_{\kappa}\right)\right),
\end{gathered}
$$

of $\operatorname{pro}^{-}\left(\text {Vect }_{F}\right)_{\kappa^{-}}\left(\right.$pro- $\left.\left(\text {Vect }_{F}\right)_{\kappa}\right)$.

Clearly, if $\kappa>\operatorname{dim} X$, then $\boldsymbol{p}_{\kappa^{-}}$is isomorphic to the rudimentary expansion $\left\lfloor 1_{X}\right\rfloor$ of $X$, while $\kappa \geq \operatorname{dim} X$ implies that $\boldsymbol{p}_{\kappa}$ is isomorphic to the rudimentary expansion $\left\lfloor 1_{X}\right\rfloor$ of $X$. We are to prove that $\boldsymbol{p}_{\kappa^{-}}: X \rightarrow \boldsymbol{X}_{\kappa^{-}}$ $\left(\boldsymbol{p}_{\kappa}: X \rightarrow \boldsymbol{X}_{\kappa}\right)$ is a $\left(V e c t_{F}\right)_{\kappa^{-}}$-expansion $\left(\left(\text {Vect }_{F}\right)_{\kappa^{-}}\right.$-expansion) of $X$ (see also Theorem 12.1 of [10]).

THEOREM 3.1. For every infinite cardinal $\kappa,\left(\text { Vect }_{F}\right)_{\kappa^{-}}$and $\left(\text {Vect }_{F}\right)_{\kappa}$ are pro-reflective subcategories of $V$ ect $_{F}$.

Proof. It suffices to verify condition (E1) for the $\boldsymbol{p}_{\kappa^{-}}$and $\boldsymbol{p}_{\kappa}$,

$$
\begin{gathered}
\boldsymbol{p}_{\kappa^{-}}=\left(p_{\lambda}\right): X \rightarrow \boldsymbol{X}_{\kappa^{-}}=\left(X_{\lambda}, p_{\lambda \lambda^{\prime}}, \Lambda_{\kappa^{-}}\right), \\
\boldsymbol{p}_{\kappa}=\left(p_{\lambda}\right): X \rightarrow \boldsymbol{X}_{\kappa}=\left(X_{\lambda}, p_{\lambda \lambda^{\prime}}, \Lambda_{\kappa}\right),
\end{gathered}
$$

constructed previously. If $\kappa>\operatorname{dim} X$, then (E1) for $\boldsymbol{p}_{\kappa^{-}}$holds trivially. Let $\kappa \leq \operatorname{dim} X$. Let $Y$ be any vectorial space (over the same field) such that $\operatorname{dim} Y<\kappa$, and let $f: X \rightarrow Y$ be a linear function. Then the kernel $N(f) \unlhd X$ and there exists a unique linear function $\tilde{f}: X / N(f) \rightarrow Y$ such that $\tilde{f} p=\bar{f}$, where $p: X \rightarrow X / N(f)$ is the quotient (linear) epimorphism. Clearly, there exists a $\lambda \in \Lambda$ such that $N(f)=Z_{\lambda}$, and thus, $X / N(f)=X_{\lambda}$ and $p=p_{\lambda}: X \rightarrow X_{\lambda}$. Since the image $R(f) \unlhd Y$, it follows that $\operatorname{dim} R(f)<\kappa$. Finally, $X \cong N(f) \oplus R(f)$ implies that $\operatorname{dim} X_{\lambda}=\operatorname{dim} R(f)<\kappa$, which shows that $\lambda \in \Lambda_{\kappa^{-}}$. In the $\kappa$-case, if $\kappa \geq \operatorname{dim} X$, then condition (E1) for $\boldsymbol{p}_{\kappa}$ holds trivially; if $\kappa<\operatorname{dim} X$, one can verify (E1) for $\boldsymbol{p}_{\kappa}$ in the same way as for $\boldsymbol{p}_{\kappa^{-}}$.

The next lemma establishes a general relationships between $\operatorname{dim} X$ and $|X|$ - the cardinality of $X$.

Lemma 3.2. Let $X$ be a vectorial space over a field $F$. Then

(i) $\operatorname{dim} X<\aleph_{0} \Rightarrow \operatorname{dim} X<|X|$.

(ii) If $|F| \leq \operatorname{dim} X$, then $\operatorname{dim} X<|X| \Rightarrow \operatorname{dim} X<\aleph_{0}$.

(iii) $\operatorname{dim} X=|X| \Rightarrow \operatorname{dim} X=\infty$.

(iv) If $|F| \leq \operatorname{dim} X$, then $\operatorname{dim} X=\infty \Rightarrow \operatorname{dim} X=|X|=\max \{\operatorname{dim} X,|F|\}$.

Proof. First of all, observe that the following inequalities generally hold true:

$(*) \quad \operatorname{dim} X \leq|X| \leq|\mathcal{F}(B)| \cdot\left|\mathcal{F}^{0}(F)\right|$ 
where $\mathcal{F}(B)$ is the set of all finite subsets of $B$ (a basis of $X$ ), and $\mathcal{F}^{0}(F)$ is the set of all ordered finite subsets of $F$. Namely, every $x \in X$ is uniquely determined by a finite subset of $B$ and an ordered finite subset of $F$. If $|B| \geq$ $\aleph_{0}\left(|F| \geq \aleph_{0}\right)$, then $|\mathcal{F}(B)|=|B|\left(\left|\mathcal{F}^{0}(F)\right|=|\mathcal{F}(F)|=|F|\right)$. Consequently, if $|B| \geq \aleph_{0}$ or $|F| \geq \aleph_{0}$, then

$$
|X| \leq|B| \cdot|F|=\max \{|B|,|F|\}
$$

holds. Since $|X| \geq \max \{|B|,|F|\}$, the second inequality in $(*)$ becomes the equality

(**) $\quad|X|=\max \{\operatorname{dim} X,|F|\}$.

whenever $|B|=\operatorname{dim} X \geq \aleph_{0}$ or $|F| \geq \aleph_{0}$. Recall that $|F| \geq 2$ holds true for every field $F$. Let us now prove the statements.

(i) Let $\operatorname{dim} X=n<\aleph_{0}$. Then $X \cong F^{n}$, and thus,

$$
\operatorname{dim} X=n<2^{n} \leq|F|^{n}=\left|F^{n}\right|=|X| .
$$

(ii) Let $\operatorname{dim} X<|X|$, where $|F| \leq \operatorname{dim} X$. Assume to the contrary, i.e., that $\operatorname{dim} X \geq \aleph_{0}$. Then $|B|=\operatorname{dim} X=\infty$, and, by (**),

$$
\operatorname{dim} X=\max \{\operatorname{dim} X,|F|\}=|X|,
$$

a contradiction. Statement (iii) follows by (i), while statement (iv) follows by (ii) and $(* *)$.

As a consequence, for instance, $\operatorname{dim}\left(\mathbb{Z}_{p}^{m}\right)=m<p^{m}=\left|\mathbb{Z}_{p}^{m}\right|, \operatorname{dim}\left(\mathbb{Z}_{2}^{\mathbb{N}}\right)=$ $\left|\mathbb{Z}_{2}^{\mathbb{N}}\right|=2^{\aleph_{0}}, \operatorname{dim}\left(\mathbb{Q}^{\mathbb{N}}\right)=\left|\mathbb{Q}^{\mathbb{N}}\right|=2^{\aleph_{0}}=\operatorname{dim}\left(\mathbb{R}_{\mathbb{Q}}\right)=|\mathbb{R}|, \operatorname{dim}\left(\mathbb{R}^{\mathbb{N}}\right)=\left|\mathbb{R}^{\mathbb{N}}\right|=$ $2^{\aleph_{0}}=\left|\mathbb{R}^{\mathbb{Q}}\right|=\operatorname{dim}\left(\mathbb{R}^{\mathbb{Q}}\right), \operatorname{dim}\left(\mathbb{R}^{\mathbb{R}}\right)=\left|\mathbb{R}^{\mathbb{R}}\right|=2^{\aleph_{1}}\left(C H\right.$ assumed, i.e., $\left.\aleph_{1}=2^{\aleph_{0}}\right)$. The well-known fact that $\operatorname{dim} X<\aleph_{0}$ is equivalent to $X \cong F^{n}$, for some $n \in \mathbb{N}$, is generalized to $\operatorname{dim} X=\infty$ in the following way.

Lemma 3.3. Let $X$ be a vectorial space over a field $F$. Then

(i) $\operatorname{dim} X=\aleph_{0} \Leftrightarrow X \cong F_{0}^{\mathbb{N}} \equiv \oplus_{i \in \mathbb{N}} F_{i}, F_{i} \equiv F$, while $\operatorname{dim} F^{\mathbb{N}}>\aleph_{0}, F^{\mathbb{N}} \equiv \Pi_{i \in \mathbb{N}} F_{i} \unrhd F_{0}^{\mathbb{N}}$

(ii) If $|F| \leq 2^{\aleph_{0}}=\aleph_{1}$ (CH assumed), then

$$
\operatorname{dim} X=2^{\aleph_{0}} \Leftrightarrow X \cong F^{\mathbb{N}}=\sqcap_{i \in \mathbb{N}} F_{i}, \quad F_{i} \equiv F .
$$

(iii) In general (GCH assumed), for each $\kappa=\aleph_{n-1} \geq \aleph_{0}, n \in \mathbb{N}$, if $|F| \leq 2^{\kappa}$, then

$$
\operatorname{dim} X=2^{\kappa} \Leftrightarrow X \cong F^{J}=\{x|x: J \rightarrow F, \quad| J \mid=\kappa\} .
$$

Proof. Lemma 3.3 is a consequence of Lemma 3.2. Nevertheless, we provide a proof with needed details.

(i) Clearly, $\operatorname{dim} X=\aleph_{0}$ if and only if, $X$ (over $F$ ) has an infinite countable basis. Since $\left\{e_{j} \mid j \in \mathbb{N}\right\}, e_{j}=\left(\delta_{i j}\right), i \in \mathbb{N}$, is a basis of $F_{0}^{\mathbb{N}} \equiv \oplus_{i \in \mathbb{N}} F_{i}$ (the canonical one), the conclusion follows. Further, since $F_{0}^{\mathbb{N}} \unlhd F^{\mathbb{N}}$ and $F^{\mathbb{N}}$ cannot have a countable basis, the proven statement implies that $\operatorname{dim} F^{\mathbb{N}}>\operatorname{dim} F_{0}^{\mathbb{N}}=$ $\aleph_{0}$. 
(ii) Let $\operatorname{dim} X=2^{\aleph_{0}}$, where $|F| \leq 2^{\aleph_{0}}$. Since $F_{0}^{\mathbb{N}} \unlhd F^{\mathbb{N}}$ and $F_{0}^{\mathbb{N}} \nsucceq F^{\mathbb{N}}$, it follows, by (i), that $\operatorname{dim}\left(F^{\mathbb{N}}\right)>\operatorname{dim}\left(F_{0}^{\mathbb{N}}\right)=\aleph_{0}$. Then $C H$ implies that $\operatorname{dim}\left(F^{\mathbb{N}}\right) \geq \aleph_{1}=2^{\aleph_{0}}$, and thus, $|F| \leq \operatorname{dim}\left(F^{\mathbb{N}}\right)=\infty$. By applying Lemma 3.2 (iv),

$$
\operatorname{dim}\left(F^{\mathbb{N}}\right)=\left|F^{\mathbb{N}}\right|=|F|^{|\mathbb{N}|} \leq\left(2^{\aleph_{0}}\right)^{\aleph_{0}}=2^{\aleph_{0} \cdot \aleph_{0}}=2^{\aleph_{0}} .
$$

holds. Consequently,

$$
\operatorname{dim}\left(F^{\mathbb{N}}\right)=2^{\aleph_{0}}=\operatorname{dim} X,
$$

which implies that $X \cong F^{\mathbb{N}}$. Conversely, let $X \cong F^{\mathbb{N}}$, where $|F| \leq 2^{\aleph_{0}}$. Then $\operatorname{dim} X=\operatorname{dim}\left(F^{\mathbb{N}}\right)$, while, as before, $|F| \leq 2^{\aleph_{0}}$ implies $\operatorname{dim}\left(F^{\mathbb{N}}\right)=2^{\aleph_{0}}$. Hence, $\operatorname{dim} X=2^{\aleph_{0}}$ too.

(iii) Firstly, notice that $|J| \leq \operatorname{dim}\left(F^{J}\right)$ holds true for every $F$ and every $J$. Namely, since $|F| \geq 2$, given any well ordering on $J$, one can easily find a set $\left\{x^{i}=\left(x_{j}^{i} \equiv x^{i}(j)\right)_{j \in J} \mid i \in I\right\} \subseteq F^{J}$ of linearly independent vectors $x^{i}$ (functions $x^{i}: J \rightarrow F$ ) such that $|I|=|J|$. (The simplest way is to choose the well ordering that yields the subset of all limit ordinals having minimal cardinalities.) Therefore,

$$
\kappa=|J| \leq \operatorname{dim}\left(F^{J}\right) \leq\left|F^{J}\right|=|F|^{|J|} \leq\left(2^{\kappa}\right)^{\kappa}=2^{\kappa \cdot \kappa}=2^{\kappa} .
$$

Hence, by $G C H$, either $\operatorname{dim}\left(F^{J}\right)=\kappa=|J|$ or $\operatorname{dim}\left(F^{J}\right)=2^{\kappa}=2^{|J|}$. If $J$ is a finite set, then the first possibility occurs. We claim that the converse also holds. In order to do it, we have to prove that $\operatorname{dim}\left(F^{J}\right)<\kappa=\infty$ implies $|J|<\kappa=\infty$, or equivalently, that for every $n \in \mathbb{N}$ and every $J_{n}$ such that $\left|J_{n}\right|=\kappa=\aleph_{n-1} \geq \aleph_{0}$, the relations

$$
\operatorname{dim}\left(F^{J_{\kappa}}\right)=2^{\left|J_{\kappa}\right|}=2^{\aleph_{n-1}}=\aleph_{n}=\left|J_{n+1}\right|
$$

hold true. The proof is by induction on $n \in \mathbb{N}$. The basic step $n=1$, i.e., $\kappa=\aleph_{0}$, is proven in the proof of claim (ii). Given an $n \in \mathbb{N}$, assume that, for all $\kappa=\aleph_{k-1}, k=1, \ldots, n, \operatorname{dim}\left(F^{J_{k}}\right)=2^{\aleph_{k-1}}=\aleph_{k}$ holds true. Let $k=n+1$, i.e., $\kappa=\aleph_{n}$. We may assume, without loss of generality, that $J_{n} \subseteq J_{n+1}$. Then, $F^{J_{n}} \unlhd F^{J_{n+1}}$ and $F^{J_{n}} \nsucceq F^{J_{n+1}}$ straightforwardly hold. Thus, $\operatorname{dim}\left(F^{J_{n}}\right)<\operatorname{dim}\left(F^{J_{n+1}}\right)$. Now, assume to the contrary, i.e., that $\operatorname{dim}\left(F^{J_{n+1}}\right)=\left|J_{n+1}\right|=\aleph_{n}$. Then, by the inductive assumption,

$$
\aleph_{n}=2^{\aleph_{n-1}}=\operatorname{dim}\left(F^{J_{n}}\right)<\operatorname{dim}\left(F^{J_{n+1}}\right)=\aleph_{n},
$$

a contradiction. Therefore, as we claimed, $\operatorname{dim}\left(F^{J}\right)=2^{\kappa}=2^{|J|}$, whenever $|J|=\kappa \geq \aleph_{0}$, Consequently, $|F| \leq \operatorname{dim}\left(F^{J}\right)=2^{\kappa}$, whenever $|F| \leq 2^{\kappa}$ and $|J|=\kappa \geq \aleph_{0}$.

Let $\kappa=\aleph_{n-1} \geq \aleph_{0}$, and let $\operatorname{dim} X=2^{\kappa}$, where $|F| \leq 2^{\kappa}$. Then, $\operatorname{dim}\left(F^{J}\right)=\operatorname{dim} X$, whenever $J$ is a set having $|J|=\kappa$, and thus, $X \cong F^{J}$. Conversely, let $X \cong F^{J}$, where $|F| \leq 2^{\kappa}$ and $|J|=\kappa \geq \aleph_{0}$. Then, as we have proven,

$$
\operatorname{dim} X=\operatorname{dim}\left(F^{J}\right)=2^{\kappa},
$$


which completes the proof.

An immediate consequence of Lemma 3.3 is, for instance, that the vectorial space $\mathbb{R}$ over $\mathbb{Q}$ is isomorphic to $\mathbb{Q}^{\mathbb{N}}$ and contains a countable-dimensional subspace isomorphic to the direct sum $\mathbb{Q}_{0}^{\mathbb{N}} \unlhd \mathbb{Q}^{\mathbb{N}}$.

LEMMA 3.4. Let $X$ be a vectorial space over a field $F$, let $X^{n}$ be the finite direct product of $n$ copies $X_{i} \equiv X, i=1, \ldots, n \in \mathbb{N}$, let $X^{\mathbb{N}}=\sqcap_{i \in \mathbb{N}} X_{i}$ be the countable infinite product of $X_{i}$ and let $X_{0}^{\mathbb{N}} \unlhd X^{\mathbb{N}}$ be the countable infinite sum of $X_{i}, i \in \mathbb{N}$. Let, for every $n \in \mathbb{N}$ and every related pair $n \leq n^{\prime}$ in $\mathbb{N}$,

$$
\pi_{n}: X^{\mathbb{N}} \rightarrow X^{n}, \quad \pi_{n n^{\prime}}: X^{n^{\prime}} \rightarrow X^{n}
$$

be the projections onto the first $n$ factors respectively, while

$$
\sigma_{n}: X^{n} \rightarrow X_{0}^{\mathbb{N}}, \quad \sigma_{n n^{\prime}}: X^{n} \rightarrow X^{n^{\prime}}
$$

be the embeddings into the first $n$ factors. Then the morphism

$$
\boldsymbol{\pi}=\left(\pi_{n}\right): X^{\mathbb{N}} \rightarrow\left(X^{n}, \pi_{n n^{\prime}}, \mathbb{N}\right)
$$

(of pro-Vect ${ }_{F}$ ) is the inverse limit in the category Vect ${ }_{F}$, while the morphism

$$
\boldsymbol{\sigma}=\left(\sigma_{n}\right):\left(X^{n}, \sigma_{n n^{\prime}}, \mathbb{N}\right) \rightarrow X_{0}^{\mathbb{N}}
$$

(of dir-Vect dis $_{\text {) }}$ the direct limit in the category $V$ ect ${ }_{F}$.

PROOF. The projections $\pi_{n n^{\prime}}$ and $\pi_{n}$ are linear functions. Since the commutativity conditions $\pi_{n n^{\prime}} \pi_{n^{\prime} n^{\prime \prime}}=\pi_{n n^{\prime \prime}}$ and $\pi_{n n^{\prime}} \pi_{n^{\prime}}=\pi_{n}, n \leq n^{\prime} \leq n^{\prime \prime}$, are obviously fulfilled, it remains to verify the universal property. Let $Y$ be a vectorial space over $F$ and let, for each $n \in \mathbb{N}$, a linear function $f_{n}: Y \rightarrow X^{n}$ be given such that, for all $n \leq n^{\prime}, \pi_{n n^{\prime}} f_{n^{\prime}}=f_{n}$. Notice that, for each $n \in \mathbb{N}$, $f_{n}=\left(f_{n}^{1}, \ldots, f_{n}^{n}\right)$, where $f_{n}^{i}: X \rightarrow X_{i}=X$ is a linear function, $i=1, \ldots, n$. Further, for every $n$ and every $i, f_{n}^{i} \equiv f^{i}$ because of $\pi_{n n^{\prime}} f_{n^{\prime}}=f_{n}$, for all $n \leq n^{\prime}$. Thus, $f_{n}=\left(f^{1}, \ldots, f^{n}\right)$ for every $n$. We can now define

$$
f: Y \rightarrow X^{\mathbb{N}}, \quad f=\left(f^{i}\right)_{i \in \mathbb{N}}
$$

that is a linear function satisfying $\pi_{n} f=f_{n}, n \in \mathbb{N}$. Suppose that $g: Y \rightarrow X^{\mathbb{N}}$ is a linear function satisfying $\pi_{n} g=\pi_{n} f=f_{n}, n \in \mathbb{N}$. Since the codomain is a direct product space, it follows that $g=\left(g^{i}\right)_{i \in \mathbb{N}}, g^{i}: Y \rightarrow X_{i}=X, i \in \mathbb{N}$. Then, for every $n \in \mathbb{N}$,

$$
\left(g^{1}, \ldots, g^{n}\right)=\pi_{n} g=\pi_{n} f=f_{n}=\left(f^{1}, \ldots, f^{n}\right) .
$$

Consequently, for each $i \in \mathbb{N}, g^{i}=f^{i}$, implying that $g=f$.

In the dual situation, $\sigma_{n n^{\prime}}$ and $\sigma_{n}$ are linear functions, and $\sigma_{n^{\prime} n^{\prime \prime}} \sigma_{n n^{\prime}}=$ $\sigma_{n n^{\prime \prime}}$ and $\sigma_{n^{\prime}} \sigma_{n n^{\prime}}=\sigma_{n}, n \leq n^{\prime} \leq n^{\prime \prime}$, hold by definitions. Let us verify the universal property. Let $Y$ be a vectorial space over $F$ and let, for each $n \in \mathbb{N}$, a linear function $u_{n}: X^{n} \rightarrow Y$ be given such that, for all $n \leq n^{\prime}$, $u_{n^{\prime}} \sigma_{n n^{\prime}}=u_{n}$. We define

$$
u: X_{0}^{\mathbb{N}} \rightarrow Y, \quad u(x)=u_{n(x)}\left(x_{1}, \ldots, x_{n(x)}\right),
$$


whenever $x=\left(x_{1}, \ldots, x_{n(x)} \neq \theta, \theta, \theta, \ldots\right) \in X_{0}^{\mathbb{N}}$, i.e., $\sigma_{n(x)}\left(x_{1}, \ldots, x_{n(x)}\right)=$ $x$. Since, for every $x \in X_{0}^{\mathbb{N}}$, there exists an $n(x) \in \mathbb{N}$ such that $x=$ $\left(x_{1}, \ldots, x_{n(x)} \neq \theta, \theta, \theta, \ldots\right)$, the function $u$ is well defined and linear, and $u \sigma_{n}=u_{n}, n \in \mathbb{N}$, obviously holds. Suppose that $v: X_{0}^{\mathbb{N}} \rightarrow Y$ is a linear function satisfying $v \sigma_{n}=u \sigma_{n}=u_{n}, n \in \mathbb{N}$. Then, for every $x=\left(x_{1}, \ldots, x_{n(x)} \neq \theta, \theta, \theta, \ldots\right) \in X_{0}^{\mathbb{N}}$,

$$
v(x)=v \sigma_{n(x)}\left(x_{1}, \ldots, x_{n(x)}\right)=u \sigma_{n(x)}\left(x_{1}, \ldots, x_{n(x)}\right)=u(x) .
$$

Therefore, $v=u$.

In the case of a finite-dimensional $X$, i.e., $X \cong F^{m}$ and $X^{\mathbb{N}}=\sqcap_{i \in \mathbb{N}}\left(F^{m}\right)_{i}$, for some $m \in\{0\} \cup \mathbb{N}$, the limit from above is isomorphic to

$$
\boldsymbol{\pi}=\left(\pi_{n}\right): \sqcap_{i \in \mathbb{N}} F_{i} \equiv F^{\mathbb{N}} \rightarrow\left(F^{n}, \pi_{n n^{\prime}}, \mathbb{N}\right) .
$$

Namely, the former one is cofinal in the later. One readily sees that the limit

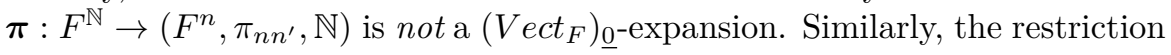

$$
\boldsymbol{\pi}^{\prime}=\left(\pi_{n}^{\prime}\right): F_{0}^{\mathbb{N}} \rightarrow\left(F^{n}, \pi_{n n^{\prime}}, \mathbb{N}\right)
$$

of the limit $\boldsymbol{\pi}: F^{\mathbb{N}} \rightarrow\left(F^{n}, \pi_{n n^{\prime}}, \mathbb{N}\right)$ (to the direct sum) is not a $\left(\text { Vect }_{F}\right)_{\underline{0}^{-}}$ expansion of $F_{0}^{\mathbb{N}}$. For instance, the linear mapping $f: F_{0}^{\mathbb{N}} \rightarrow F$ defined on the (canonical) basis $\left(e_{i}=\left(\delta_{j i}\right)_{j \in \mathbb{N}}\right)_{i \in \mathbb{N}}$ of $F_{0}^{\mathbb{N}}$ by $f\left(e_{i}\right)=1, i \in \mathbb{N}$, does not admit a factorization through any $\pi_{n}^{\prime}: F_{0}^{\mathbb{N}} \rightarrow F^{n}$.

THEOREM 3.5. For every infinite cardinal $\kappa \geq \aleph_{0}, S h_{\kappa^{-}}\left(F^{\mathbb{N}}\right) \neq$ $S h_{\kappa^{-}}\left(F_{0}^{\mathbb{N}}\right)$ and $S h_{\kappa}\left(F^{\mathbb{N}}\right) \neq S h_{\kappa}\left(F_{0}^{\mathbb{N}}\right)$.

Proof. According to Lemma 3.3, the only non-trivial cases to prove are $S h_{\underline{0}}\left(F^{\mathbb{N}}\right) \neq S h_{0}\left(F_{0}^{\mathbb{N}}\right)$ and $S h_{\aleph_{0}}\left(F^{\mathbb{N}}\right) \neq S h_{\aleph_{0}}\left(F_{0}^{\mathbb{N}}\right)$. It suffices to show that $S h_{\underline{0}}\left(F^{\mathbb{N}}\right) \neq S h_{\underline{0}}\left(F_{0}^{\mathbb{N}}\right)$ (because $S h_{\kappa}(X)=S h_{\kappa}(Y)$ implies $S h_{\kappa^{-}}(X)=$ $S h_{\kappa^{-}}(Y \overline{)})$. Let

$$
\begin{aligned}
& \boldsymbol{p}_{\underline{0}}=\left(p_{\lambda}\right): F^{\mathbb{N}} \rightarrow\left(X_{\lambda}, p_{\lambda \lambda^{\prime}}, \Lambda_{\underline{0}}\right) \equiv \boldsymbol{X}_{\underline{0}} \text { and } \\
& \boldsymbol{q}_{\underline{0}}=\left(q_{\mu}\right): F_{0}^{\mathbb{N}} \rightarrow\left(Y_{\mu}, q_{\mu \mu^{\prime}}, M_{\underline{0}}\right) \equiv \boldsymbol{Y}_{\underline{0}}
\end{aligned}
$$

be $\left(\text { Vect } t_{F}\right)_{0}$-expansions (constructed as above) of $F^{\mathbb{N}}$ and $F_{0}^{\mathbb{N}}$ respectively. Recall that, for each $\lambda \in \Lambda_{0}, X_{\lambda}=F^{\mathbb{N}} / Z_{\lambda}$, where $\operatorname{dim} Z_{\lambda}=\operatorname{dim} F^{\mathbb{N}}>\aleph_{0}$ (thus, $Z_{\lambda} \cong F^{\mathbb{N}}$ ) and $\operatorname{dim} X_{\lambda}<\aleph_{0}$ (thus, $X_{\lambda} \cong F^{n(\lambda)}$ ), while, for each $\mu \in M_{0}, Y_{\mu}=F_{0}^{\mathbb{N}} / W_{\mu}$, where $\operatorname{dim} W_{\mu}=\operatorname{dim} F_{0}^{\mathbb{N}}=\aleph_{0}$ (thus, $W_{\mu} \cong F_{0}^{\mathbb{N}}$ ) and $\operatorname{dim} Y_{\mu}<\aleph_{0}$ (thus, $Y_{\mu} \cong F^{n(\mu)}$ ). Further, $F^{\mathbb{N}} \cong F_{0}^{\mathbb{N}} \oplus F^{\mathbb{N}}$. Further, $\lambda<\lambda^{\prime}$ and $\mu<\mu^{\prime}$ imply the strictly increasing finite dimension. Let us assume to the contrary, i.e., that there exists an isomorphism

$$
\boldsymbol{f}=\left[\left(\varphi, f_{\mu}\right)\right]: \boldsymbol{X}_{\underline{0}} \rightarrow \boldsymbol{Y}_{\underline{0}}
$$

of $\operatorname{pro}\left(\operatorname{Vect}_{F}\right)_{\underline{0}}$, and thus,

$$
\boldsymbol{p}_{\underline{0}} \boldsymbol{f}=\left(p_{\varphi(\mu)} f_{\mu}\right): F^{\mathbb{N}} \rightarrow \boldsymbol{Y}_{\underline{0}}
$$


is a $\left(V e c t_{F}\right)_{0^{-}}$expansion of $F^{\mathbb{N}}$. Since the (finite) dimension of terms in systems is increasing and unbounded, $\varphi\left[M_{0}\right] \subseteq \Lambda_{\underline{0}}$ has to be cofinal. Observe that $\left|\Lambda_{0}\right|>\left|M_{0}\right|=\infty$ implies that the function $\varphi$ misses almost all (with respect to the cardinality $\left.\left|\Lambda_{\underline{0}}\right|\right) \lambda \in \Lambda_{\underline{0}}$. Now, given a linear $g: F^{\mathbb{N}} \rightarrow Z, Z \cong F^{k}$ for some $k \in \mathbb{N}$, that factorizes through a $p_{\lambda}, \lambda \in \Lambda_{\underline{0}} \backslash \varphi\left[M_{\underline{0}}\right]$, an isomorphism $h_{g}: X_{\lambda} \rightarrow X_{\varphi(\mu)}$ (essentially depending on $g$ ) is needed to obtain the unique factorization (E1) of $f$ through $\boldsymbol{p}_{\underline{0}} \boldsymbol{f}$. It is clear that the family of all such linear functions $g$ does not admit a certain morphism of the considered inverse systems. Consequently, $\boldsymbol{p}_{\underline{0}} \boldsymbol{f}$ cannot be an expansion of $F^{\mathbb{N}}$ - a contradiction.

REMARK 3.6. According to Lemma 3.3, the above results can be (algebraically) generalized to arbitrary direct products and sums in the following way: Let $F$ be a field, let $J$ be a set of cardinality $|J|=\kappa \geq \aleph_{0}$ and let $F_{j}=F$ for every $j \in J$. Let us well order $J$ by choosing the well ordering $\leq$ that yields the subset of all limit ordinals having minimal cardinality. Consider the direct product

$$
\sqcap_{j \in J} F_{j}=\{x \mid x: J \rightarrow F\} \equiv F^{J},
$$

endowed with the coordinatewise operations, to be a vectorial space over $F$. Put

$$
J_{\kappa^{-}}=\{j||[\min J, j] \mid<\kappa\} \subseteq J .
$$

Then the subset

$$
\left\{x \mid\left(\exists j_{x} \in J_{\kappa^{-}}\right)\left(j>j_{x} \Rightarrow x(j)=0\right)\right\} \subseteq F^{J}
$$

is a vectorial subspace, denoted by $\oplus_{j \in J}^{\kappa^{-}} F_{j} \equiv F_{\kappa^{-}}^{J}$ and called the direct $\kappa$-sum of $F_{j}=F$. (In the case of $\kappa=\aleph_{0}$ and $(J, \leq) \cong \mathbb{N}$, the direct $\aleph_{0}$-sum is the ordinary direct sun.) Put $F^{j} \equiv \sqcap_{j^{\prime} \leq j} F_{j^{\prime}}$. Then

$$
\boldsymbol{\pi}=\left(\pi_{j}\right): F^{J} \rightarrow\left(F^{j}, \pi_{j j^{\prime}}, J\right)
$$

$\left(\pi_{j j^{\prime}}\right.$ and $\pi_{j}$ are the appropriate projections) is the inverse limit in $V e c t_{F}$, while

$$
\boldsymbol{\sigma}=\left(\sigma_{j}\right):\left(F^{j}, \sigma_{j j^{\prime}}, J\right) \rightarrow F_{\kappa^{-}}^{J}
$$

$\left(\sigma_{j j^{\prime}}\right.$ and $\sigma_{j}$ are the appropriate embeddings) is the direct limit in $V e c t_{F}$. Again, nor $\boldsymbol{\pi}=\left(\pi_{j}\right): F^{J} \rightarrow\left(F^{j}, \pi_{j j^{\prime}}, J\right)$ is a $\left(V e c t_{F}\right)_{\kappa^{-}}$-expansion of $F^{J}$ neither its restriction $\boldsymbol{\pi}^{\prime}=\left(\pi_{j}^{\prime}\right): F_{\kappa^{-}}^{J} \rightarrow\left(F^{j}, \pi_{j j^{\prime}}, J\right)$ is a $\left(V_{e c t}\right)_{\kappa^{-}}$-expansion of $F_{\kappa^{-}}^{J}$. Observe that (by Lemma 3.3 and $G C H$ assumed), if $|F| \leq 2^{\kappa}$, then $\operatorname{dim}\left(F^{J}\right)=\left|F^{J}\right|=2^{\kappa}$, while $\operatorname{dim} F_{\kappa^{-}}^{J}=\kappa$. Namely, if $n \in \mathbb{N},|F| \leq$ $2^{\aleph_{n-1}}=\aleph_{n}$ and $\left|J_{n}\right|=\aleph_{n-1}$, then the direct product $\Pi_{j \in J_{n}} F_{j} \equiv F^{j_{n}}$ is isomorphic to the direct $\kappa$-sum $F_{\kappa^{--}}^{J_{n+1}} \unlhd \sqcap_{j \in J_{n+1}} F_{j} \equiv F^{j_{n+1}}$, where $J_{n+1} \supseteq J_{n}$ and $\left|J_{n+1}\right|=\kappa=\aleph_{n}$. 
Notice that a $\left(V e c t_{F}\right)_{\kappa^{-}}$-expansion and a $\left(V e c t_{F}\right)_{\kappa^{-}}$expansion of $X$ depend on $\operatorname{dim} X$ only. Therefore, though there exist the various (quotient) shapes $S h_{\kappa^{-}}(X)$ and $S h_{\kappa}(X)$ of a vectorial space $X$ and the shape morphism sets are richer than the linear function sets, all the (quotient) shape classifications on $V e c t_{F}$ and the isomorphisms classification coincide. Namely, the appropriate sets of subspaces of $X$ are needed only. So the conclusion for $V e c t_{F}$ is quite similar to that for the category Set (see [10], Theorem 4.7). Thus the classification result is as follows.

THEOREM 3.7. For every pair of vectorial spaces $X, Y$ over a field $F$, the following statements are equivalent:

(i) $S h_{0}(X)=S h_{0}(Y)$

(ii) $\left(\forall \kappa \geq \aleph_{0}\right) S h_{\kappa^{-}}(X)=S h_{\kappa^{-}}(Y)$;

(iii) $\left(\forall \kappa \geq \aleph_{0}\right) S h_{\kappa}(X)=S h_{\kappa}(Y)$;

(iv) $X \cong Y\left(\right.$ in $_{\text {Vect }}$, i.e., linearly isomorphic);

(v) $\operatorname{dim} X=\operatorname{dim} Y$.

Proof. According to well known facts (see also the proof of Theorem 3.5 ), it suffices to verify that (i) implies (v) in the infinite-dimensional case. Let $X$ and $Y$ be infinite-dimensional vectorial spaces over the same field $F$, and let $S h_{0}(X)=S h_{0}(Y)$. This means that the inverse systems $\boldsymbol{X}_{0}$ and $\boldsymbol{Y}_{0}$ in the canonical expansions $\boldsymbol{p}_{0}: X \rightarrow \boldsymbol{X}_{\underline{0}}$ and $\boldsymbol{q}_{0}: Y \rightarrow \boldsymbol{Y}_{\underline{0}}$ of $X$ and $Y$, respectively, are isomorphic objects of pro- $\left(V e c t_{F}\right)_{\underline{0}}$. Recall that this construction includes every subspace of the maximal dimension (which is infinite) having a finite codimension. Thus, it fully and only depends on the dimension of a space. Consequently, $\boldsymbol{X}_{\underline{0}} \cong \boldsymbol{Y}_{\underline{0}}$ in $\operatorname{pro}\left(\text { Vect }_{F}\right)_{\underline{0}}$ implies that $\operatorname{dim} X=\operatorname{dim} Y$ must hold.

We finish this section with the following lemma (needed in the sequel).

LEMma 3.8. Let $X$ be a vectorial space such that $\operatorname{dim} X \geq \kappa \geq \aleph_{0}$ ( $\operatorname{dim} X>\kappa \geq \aleph_{0}$ ), and let $W_{1}$ and $W_{2}$ be non-trivial subspaces of $X$, $W_{1,2} \unlhd X,\{\theta\} \neq W_{1,2} \neq X$.

(i) If $\operatorname{dim} W_{1}<\kappa(\leq \kappa)$, then, for every basis $B$ of $X$, there exists a proper subset $B^{\prime} \subseteq B$ such that $\left|B^{\prime}\right|<\kappa(\leq \kappa), W_{1} \unlhd L_{B^{\prime}}$ and $B \backslash B^{\prime}$ contains almost all the basis' vectors. i.e., $\left|B \backslash B^{\prime}\right|=|B|=\operatorname{dim} X$. Further, there exists a direct complement $Z_{1}$ of $W_{1}$ in $X$, i.e., $X=W_{1} \oplus Z_{1}$, such that $L_{B \backslash B^{\prime}} \unlhd Z_{1}$ and $\operatorname{dim} Z_{1}=\operatorname{dim} X$.

(ii) If $\operatorname{dim} W_{2}=\operatorname{dim} X$ and $\operatorname{dim} X / W_{2}<\kappa(\leq \kappa)$, then, for every basis $B$ of $X$, there exists a proper subset $B^{\prime \prime} \subseteq B$ such that $\left|B^{\prime \prime}\right|=|B|=$ $\operatorname{dim} X, W_{2} \unlhd L_{B^{\prime \prime}}$ and $B^{\prime \prime}$ contains almost all the basis'vectors. i.e., $\left|B \backslash B^{\prime \prime}\right|<\kappa(\leq \kappa)$. Further, there exists a direct complement $Y_{2}$ of $W_{2}$ in $X$, i.e., $X=Y_{2} \oplus W_{2}$, such that $L_{B \backslash B^{\prime \prime}} \unlhd Y_{2}$ and $\operatorname{dim} Y_{2}<\kappa$ $(\leq \kappa)$. 
(iii) If $\operatorname{dim} W_{1}=\operatorname{dim} X=\operatorname{dim} W_{2}$ and $\max \left\{\operatorname{dim} X / W_{1}, \operatorname{dim} X / W_{2}\right\}<\kappa$ $(\leq \kappa)$, then $\operatorname{dim}\left(W_{1} \cap W_{2}\right)=\operatorname{dim} X$ and $\operatorname{dim} X /\left(W_{1} \cap W_{2}\right)<\kappa(\leq \kappa)$.

Proof. (i) Let $W_{1} \unlhd X$ such that $\operatorname{dim} X \geq \kappa \geq \aleph_{0}$ and $\operatorname{dim} W_{1}<\kappa$. Let $B=\left\{e_{j} \mid j \in J\right\}$ be a basis of $X$. Choose a basis $C_{1}=\left\{u_{\tau} \mid \tau \in T_{1}\right\}$ of $W_{1}$. Then $\left|T_{1}\right|=\operatorname{dim} W_{1}<\kappa$. Clearly, every $u_{\tau} \in C_{1}$ is a finite linear combination of some elements $e_{j}$ of $B$ (with coefficients from the field). Let

$$
B^{\prime}=\left\{e_{j^{\prime}} \mid j^{\prime} \in J^{\prime} \subseteq J\right\} \subseteq B
$$

be the subset of all such elements $e_{j}$ of $B$ (for all $\tau \in T_{1}$ ). Then

$$
\left|B^{\prime}\right|=\left|J^{\prime}\right| \leq\left|T_{1}\right| \cdot \aleph_{0}<\kappa \cdot \aleph_{0}=\kappa \leq \operatorname{dim} X,
$$

$B^{\prime} \subseteq B$ is a proper subset and $W_{1}=\left[C_{1}\right] \unlhd L_{B^{\prime}}$. Further, $B \backslash B^{\prime}$ contains almost all the basis' vectors. Namely, $\left|B^{\prime}\right|<\kappa \geq \aleph_{0}$, while

$$
\kappa \leq \operatorname{dim} X=|B|=|B| \backslash\left|B^{\prime}\right|=\left|B \backslash B^{\prime}\right| .
$$

Since $W_{1} \unlhd L\left(B^{\prime}\right)$, it follows that

$$
X=L_{B^{\prime}} \oplus L_{B \backslash B^{\prime}}=W_{1} \oplus W^{\prime} \oplus L_{B \backslash B^{\prime}},
$$

and we may put $Z_{1}=W^{\prime} \oplus L_{B \backslash B^{\prime}}$. Finally,

$$
\operatorname{dim} X \geq \operatorname{dim} Z_{1} \geq\left|B \backslash B^{\prime}\right|=\operatorname{dim} X
$$

implies that $\operatorname{dim} Z_{1}=\operatorname{dim} X$. In the case of $\operatorname{dim} X>\kappa$ and $\operatorname{dim} W_{1} \leq \kappa$, the proof goes in the same manner.

(ii) Let $W_{2} \unlhd X$ such that $\operatorname{dim} X \geq \kappa \geq \aleph_{0}$, $\operatorname{dim} W_{2}=\operatorname{dim} X$ and $\operatorname{dim} X / W_{2}<\kappa$. Let $B=\left\{e_{j} \mid j \in J\right\}$ be a basis of $X$. Choose a basis $C_{2}=\left\{u_{\tau} \mid \tau \in T_{2}\right\}$ of $W_{2}$. Then $\left|T_{2}\right|=\operatorname{dim} W=\operatorname{dim} X=|J|$. As before, every $u_{\tau}$ is a finite linear combination of some elements $e_{j}$ of $B$. Put

$$
B^{\prime \prime}=\left\{e_{j^{\prime \prime}} \mid j^{\prime \prime} \in J^{\prime \prime} \subseteq J\right\} \subseteq B
$$

to be the subset of all such elements $e_{j}$ of $B$ (for all $\tau \in T_{2}$ ). Since $W_{2} \unlhd X$ is a non-trivial subspace, it follows that $B^{\prime \prime} \subseteq B$ is a proper subset,

$$
\left|B^{\prime \prime}\right|=\left|J^{\prime \prime}\right| \leq\left|T_{2}\right| \cdot \aleph_{0}=|J| \cdot \aleph_{0}=|J|=|B|
$$

and $W_{2}=\left[C_{2}\right] \unlhd L_{B^{\prime \prime}}$. Further,

$$
\left|B \backslash B^{\prime \prime}\right|=|B| \backslash\left|B^{\prime \prime}\right|<|B|
$$

because of $\operatorname{dim} X / W<\kappa \leq \operatorname{dim} X=\operatorname{dim} W$. Since $W_{2} \unlhd L_{B^{\prime \prime}}$, it follows that

$$
X=L_{B \backslash B^{\prime \prime}} \oplus L_{B^{\prime \prime}}=L_{B \backslash B^{\prime \prime}} \oplus W^{\prime \prime} \oplus W_{2},
$$

and we may put $Y_{2}=L_{B \backslash B^{\prime \prime}} \oplus W^{\prime \prime}$. Finally, $X=Y_{2} \oplus W_{2}$ implies that

$$
\operatorname{dim} Y_{2}=\operatorname{dim} X / W_{2}<\kappa .
$$

In the case of $\operatorname{dim} X>\kappa$ and $\operatorname{dim} X / W_{2} \leq \kappa$, the proof goes in the same way.

(iii) Let $W_{1}, W_{2} \unlhd X$ such that $\operatorname{dim} X \geq \kappa \geq \aleph_{0}, \operatorname{dim} W_{1}=\operatorname{dim} X=$ $\operatorname{dim} W_{2}$ and $\max \left\{\operatorname{dim} X / W_{1}, \operatorname{dim} X / W_{2}\right\}<\kappa$. If $W_{1} \unlhd W_{2}$ or $W_{2} \unlhd W_{1}$, 
then the statement holds trivially. Assume that $W_{1} \cap W_{2} \neq W_{1,2}$. Then there exits a direct complement $Y_{1} \unlhd X$ of $W_{1}$, i.e., $X=Y_{1} \oplus W_{1}$, such that $Y_{1} \not W_{2}$. Put $Y_{1}=Y_{1}^{\prime} \oplus Y_{1}^{\prime \prime}$, where $Y_{1}^{\prime} \cap W_{2}=\{\theta\}$ and $Y_{1}^{\prime \prime} \unlhd W_{2}$. Then

$$
Y_{1} \oplus\left(W_{1} \cap W_{2}\right)=\left(Y_{1} \oplus W_{1}\right) \cap\left(Y_{1}^{\prime \prime} \oplus W_{2}\right)=X \cap\left(Y_{1}^{\prime} \oplus W_{2}\right)=Y_{1}^{\prime} \oplus W_{2} .
$$

Since $\operatorname{dim} Y_{1}^{\prime} \leq \operatorname{dim} Y_{1}<\kappa \leq \operatorname{dim} W_{2}=\operatorname{dim} X \geq \aleph_{0}$, we infer that $\operatorname{dim}\left(W_{1} \cap\right.$ $\left.W_{2}\right)=\operatorname{dim} X$. Let $Y_{2} \unlhd X$ be a direct complement of $W_{2}$. Choose arbitrary $Z_{1} \cong Y_{1}, Z_{2} \cong W_{1} \cap W_{2}$ and $Z_{3} \cong Y_{2}$, and put $Z=Z_{1} \oplus Z_{2} \oplus Z_{3}$. Since, as we have seen, $Y_{1} \oplus\left(W_{1} \cap W_{2}\right)=Y_{1}^{\prime} \oplus W_{2}$ in $X$ and $Y_{1}^{\prime} \unlhd Y_{1}$, it follows that there exists a subspace $Z^{\prime} \unlhd Z$ such that $X \cong Z^{\prime}$ and $W_{1} \cap W_{2} \unlhd Z^{\prime}$. Consequently,

$$
\begin{gathered}
\operatorname{dim} X /\left(W_{1} \cap W_{2}\right)=\operatorname{dim} Z^{\prime} /\left(W_{1} \cap W_{2}\right) \leq \operatorname{dim} Z /\left(W_{1} \cap W_{2}\right) \\
=\operatorname{dim} Y_{1}+\operatorname{dim} Y_{2}=\operatorname{dim} X / W_{1}+\operatorname{dim} X / W_{2}<\kappa+\kappa=\kappa,
\end{gathered}
$$

that completes the proof of the statement (iii) in this case. In the case of $\operatorname{dim} X>\kappa$ and $\operatorname{dim} X / W_{2} \leq \kappa$, the proof is quite similar.

Observe that there is no finite-dimensional analogue of Lemma 3.8.

\section{The QUOTIENT SHAPES OF TOPOLOGICAL VECTORIAL SPACES}

In this section, we consider the quotient shapes in the category of topological vectorial spaces over a topologized field $F$ and their continuous linear functions - $T V e c t_{F}$, and, especially (for a few particular fields), the quotient shapes in the category of normed vectorial spaces - NVect ${ }_{F}$, which is a full subcategory of $T V$ ect $_{F}$. Recall that a topological vectorial space $X$ over (a topologized field) $F$ is an ordered pair $(V, \mathcal{T})$, where $V$ is a vectorial space over $F$ and $\mathcal{T}$ is a topology on $V$ such that the operations

$$
\begin{gathered}
+: X \times X \rightarrow X, \quad+(x, y)=x+y, \quad \text { and } \\
\cdot: F \times X \rightarrow X, \quad \cdot(\alpha, x)=\alpha x
\end{gathered}
$$

are continuous with respect to the direct product topologies on $X \times X$ and $F \times X$ respectively. Usually or very often, one asks for the additional condition that $\mathcal{T}$ has to be a Hausdorff $\left(T_{2}\right)$ topology, and in that case the topology on $F$ has to be Hausdorff too. In such a case, let the special notation for the corresponding category be $T_{2} V e c t_{F}$.

A normed vectorial space $X$ over $F \in\{\mathbb{Q}, \mathbb{R}, \mathbb{C}\}$ is an ordered pair $(V,\|\cdot\|)$, also denoted by $(X,\|\cdot\|)$, where $V$ is a vectorial space over $F$ and $\|\cdot\|: V \rightarrow \mathbb{R}$ is a norm (satisfying the well-known conditions). Equivalently, a normed vectorial space $X$ over $F \in\{\mathbb{Q}, \mathbb{R}, \mathbb{C}\}$ is a topological vectorial space $\left(V, \mathcal{T}_{d}\right)$, over $F$ carrying the euclidean topology, such that $\mathcal{T}_{d}$ is the metric topology induced by a given norm $\|\cdot\|$ on $V$, i.e.,

$$
(\forall x, y \in X) d(x, y)=\|x-y\| \text {. }
$$


Usually, one takes $F \in\{\mathbb{R}, \mathbb{C}\}$ only, and speaks about a real or complex normed space. However, in our general consideration, there is no reason for excluding the case $F=\mathbb{Q}$. (Clearly, a normed $X$ over $\mathbb{Q}$ cannot be a Banach space.)

Let $\kappa \geq \aleph_{0}$ be an infinite cardinal, and let $\left(T V e c t_{F}\right)_{\kappa^{-}}$and $\left(N V e c t_{F}\right)_{\kappa^{-}}$ $\left(\left(T V e c t_{F}\right)_{\kappa}\right.$ and $\left.\left(N V e c t_{F}\right)_{\kappa}\right)$ be the full subcategory of $T V e c t_{F}$ and $N V e c t_{F}$, respectively, determined by all the objects having the algebraic dimension less than (less than or equal to) $\kappa$. We shall begin with the special case.

4.1. The normed case. As we mentioned before, in this subsection we assume that $F \in\{\mathbb{Q}, \mathbb{R}, \mathbb{C}\}$ carrying the euclidean topology. The category $N V$ ect $_{F}$ of all normed vectorial spaces and their continuous linear functions is a very special full subcategory of $T V$ ect $_{F}$. Recall that a (normed) quotient space of a normed vectorial space $X$ asks for a closed subspace $Z \unlhd X$. Then the "quotient" norm on the quotient vectorial space $X / Z$ is defined by

$$
\|[x]\|=\inf \{\|x+z\| \mid z \in Z\} .
$$

First of all, we have to prove that every infinite-dimensional normed vectorial space $X$ contains "sufficiently many" proper closed subspaces $Z$ such that $\operatorname{dim} Z=\operatorname{dim} X$ and the dimension of an (every) algebraic direct complement is less than $\operatorname{dim} X$, i.e., $\operatorname{dim}(X / Z)<\operatorname{dim} X$.

LEMMA 4.1. Let $X$ be an infinite-dimensional normed vectorial space, let $Y$ be a topological vectorial space (over the same field) with $\operatorname{dim} Y<\operatorname{dim} X$ and let $f: X \rightarrow Y$ be a continuous non-null linear function. Then the kernel $N(f)$ is a proper closed subspace of $X, \operatorname{dim} N(f)=\operatorname{dim} X$ and $0<$ $\operatorname{dim}(X / N(f))<\operatorname{dim} X$. If, especially, $Y$ is $\kappa$-dimensional and $\kappa \geq \aleph_{0}$, then $\operatorname{dim}(X / N(f)) \leq \kappa$

Proof. Observe that, algebraically, $X=N(f) \oplus X^{\prime}, X^{\prime} \cong R(f)$, and $\operatorname{dim} X^{\prime}=\operatorname{dim} R(f) \leq \operatorname{dim} Y<\operatorname{dim} X$ hold. By Lemma 3.8 (i), for every basis $B$ of $X$, there is a subset $B^{\prime} \subseteq B$ such that $X^{\prime} \unlhd L\left(B^{\prime}\right)$ and $\left|B \backslash B^{\prime}\right|=$ $|B|$. Since $\operatorname{dim} Y<\operatorname{dim} X$, it follows that $\left|B^{\prime}\right|<\operatorname{dim} X=|B|$. Therefore, $\operatorname{dim} N(f)=|B|=\operatorname{dim} X$ and $\operatorname{dim}(X / N(f)) \leq\left|B^{\prime}\right|<\operatorname{dim} X$. Further, if $\operatorname{dim} Y=\kappa \geq \aleph_{0}$, then $\operatorname{dim}(X / N(f))=\operatorname{dim} R(f) \leq \operatorname{dim} Y \leq \kappa$. Finally, $f \neq c_{\theta}$ implies that $N(f)$ is a proper subspace of $X$, and $N(f)$ is closed because $f$ is continuous.

By Hahn-Banach theorem ([7], Section 6.5), there are "sufficiently many" continuous linear functions needed in Lemma 4.1. This further implies that there are "sufficiently many" closed subspaces of a normed vectorial space $X$ having dimension $\operatorname{dim} X$ and codimension less than $\operatorname{dim} X$. For instance, if $W \unlhd X$ is closed and $Y \unlhd X$ is finite-dimensional such that $Y \cap W=\{\theta\}$, then there exists a closed subspace $Z \unlhd X$ such that $X=Y \oplus Z$ and $W \unlhd Z$ ([7], Section 6.5, Exercise 12). 
THEOREM 4.2. For each cardinal $\kappa \geq \aleph_{0}$, the categories $\left(N V e c t_{F}\right)_{\kappa^{-}}$and $\left(N V e c t_{F}\right)_{\kappa}$ are pro-reflective subcategories of NVect ${ }_{F}$.

Proof. Let $X \in O b\left(N V e c t_{F}\right)$ and let $\kappa \geq \aleph_{0}$ be an infinite cardinal. If $\operatorname{dim} X<\kappa(\operatorname{dim} X \leq \kappa)$, then the identity $\left\lfloor 1_{X}\right\rfloor: X \rightarrow\lfloor X\rfloor$ is a rudimentary $\left(N V e c t_{F}\right)_{\kappa^{-}}$-expansion $\left(\left(N V e c t_{F}\right)_{\kappa^{-}}\right.$expansion $)$of $X$. Let $\operatorname{dim} X \geq \kappa$ $(\operatorname{dim} X>\kappa)$. Let $\left\{Z_{\lambda} \mid \lambda \in \Lambda\right\}$ be the set of all closed subspaces $Z_{\lambda} \unlhd X$ such that $\operatorname{dim} Z_{\lambda}=\operatorname{dim} X$ and $\operatorname{dim}\left(X / Z_{\lambda}\right)<\operatorname{dim} X$. Let us order the set $\Lambda$ by putting

$$
\lambda \leq \lambda^{\prime} \Leftrightarrow Z_{\lambda^{\prime}} \unlhd Z_{\lambda} .
$$

Observe that $(\Lambda, \leq)$ is directed. Indeed, for every pair $\lambda, \lambda^{\prime} \in \Lambda, Z_{\lambda} \cap Z_{\lambda^{\prime}} \unlhd X$ is a closed subspace, $Z_{\lambda} \cap Z_{\lambda^{\prime}} \unlhd Z_{\lambda}, Z_{\lambda} \cap Z_{\lambda^{\prime}} \unlhd Z_{\lambda^{\prime}}$ and, by Lemma 3.8 (iii), $\operatorname{dim}\left(Z_{\lambda} \cap Z_{\lambda^{\prime}}\right)=\operatorname{dim} X$ and $\operatorname{dim}\left(X /\left(Z_{\lambda} \cap Z_{\lambda^{\prime}}\right)\right)<\operatorname{dim} X$. Now, for each $\lambda \in \Lambda$, put $X_{\lambda}=X / Z_{\lambda}$ to be the normed quotient vectorial space. Let $p_{\lambda}: X \rightarrow X_{\lambda}$ and $p_{\lambda \lambda^{\prime}}: X_{\lambda^{\prime}} \rightarrow X_{\lambda}, \lambda \leq \lambda^{\prime}$, be the quotient functions, that are epimorphic, linear, continuous and open ([7], Section 8.2). In this way we have constructed an inverse system

$$
\boldsymbol{X}=\left(X_{\lambda}, p_{\lambda \lambda^{\prime}}, \Lambda\right)
$$

in $N V e c t_{F}$ and a morphism

$$
\boldsymbol{p}=\left(p_{\lambda}\right): X \rightarrow \boldsymbol{X}
$$

of pro-NVect $F$. Denote by $\left(\Lambda_{\kappa}, \leq\right)\left(\left(\Lambda_{\kappa}, \leq\right)\right)$ the ordered subset of $(\Lambda, \leq)$ consisting of all $\lambda \in \Lambda$ such that $\operatorname{dim}\left(X / Z_{\lambda}\right)<\kappa\left(\operatorname{dim}\left(X / Z_{\lambda}\right) \leq \kappa\right)$. By Lemma 4.1, $\Lambda_{\kappa^{-}}$is not empty. Moreover, it is directed. Namely, if in addition,

$$
\begin{gathered}
\max \left\{\operatorname{dim}\left(X / Z_{\lambda}\right), \operatorname{dim}\left(X / Z_{\lambda^{\prime}}\right)\right\}<\kappa \leq \operatorname{dim} X \\
\left(\max \left\{\operatorname{dim}\left(X / Z_{\lambda}\right), \operatorname{dim}\left(X / Z_{\lambda^{\prime}}\right)\right\} \leq \kappa<\operatorname{dim} X\right),
\end{gathered}
$$

then by Lemma 3.8 (iii),

$$
\operatorname{dim}\left(X /\left(Z_{\lambda} \cap Z_{\lambda^{\prime}}\right)\right)<\kappa \quad\left(\operatorname{dim}\left(X /\left(Z_{\lambda} \cap Z_{\lambda^{\prime}}\right)\right) \leq \kappa\right) .
$$

Finally, by the general theory and our construction, the restriction

$$
\begin{gathered}
\boldsymbol{p}_{\kappa^{-}}=\left(p_{\lambda}\right): X \rightarrow \boldsymbol{X}_{\kappa^{-}}=\left(X_{\lambda}, p_{\lambda \lambda^{\prime}}, \Lambda_{\kappa^{-}}\right) \\
\left(\boldsymbol{p}_{\kappa}=\left(p_{\lambda}\right): X \rightarrow \boldsymbol{X}_{\kappa}=\left(X_{\lambda}, p_{\lambda \lambda^{\prime}}, \Lambda_{\kappa}\right)\right)
\end{gathered}
$$

$\boldsymbol{p}$ is an $\left(N V e c t_{F}\right)_{\kappa^{-}}$-expansion $\left(\left(N V e c t_{F}\right)_{\kappa^{-}}\right.$expansion $)$of $X$.

The following corollary is a consequence of some general facts.

Corollary 4.3. Let $X, Y \in O b\left(N V e c t_{F}\right)$ such that $S h_{\kappa^{-}}(X)=$ $S h_{\kappa^{-}}(Y)$, for some $\kappa \leq \operatorname{dim} X$. Then,

(i) $\operatorname{dim} X<\infty \Rightarrow Y \cong X$ (in NVect $\left.{ }_{F}\right)$;

(ii) $\operatorname{dim} X=\infty \Rightarrow \operatorname{dim} Y=\operatorname{dim} X$. 
Proof. Clearly, it suffices to prove the statements in the case $\kappa=\aleph_{0}$.

(i) Since $X$ is finite-dimensional, $\left\lfloor 1_{X}\right\rfloor: X \rightarrow\lfloor X\rfloor$ is a rudimentary finitedimensional (and a rudimentary $\kappa^{-}$- and $\kappa$-, for every $\kappa \geq \aleph_{0}$ ) expansion of $X$. Consequently, $S h_{\underline{0}}(X)=S h_{\underline{0}}(Y)$ implies that $Y$ admits a rudimentary finite-dimensional expansion $\lfloor q\rfloor: Y \rightarrow\lfloor X\rfloor$. Therefore, $q: Y \rightarrow X$ is an isomorphism of $N V e c t_{F}$.

(ii) Let $\boldsymbol{p}_{\underline{0}}=\left(p_{\lambda}\right): X \rightarrow \boldsymbol{X}_{\underline{0}}=\left(X_{\lambda}, p_{\lambda \lambda^{\prime}}, \Lambda_{\underline{0}}\right)$ and $\boldsymbol{q}_{\underline{0}}=\left(q_{\mu}\right): Y \rightarrow$ $\boldsymbol{Y}_{0}=\left(Y_{\mu}, q_{\mu \mu^{\prime}}, M_{0}\right)$ be appropriate expansions of $X$ and $Y$ respectively. Then $\boldsymbol{X}_{\underline{0}}^{-} \cong \boldsymbol{Y}_{\underline{0}}$ in pro- $\left(N \text { Nect }_{F}\right)_{\underline{0}}$. Consequently, by forgetting the normed structure, $\boldsymbol{X}_{\underline{0}}^{-} \cong \boldsymbol{Y}_{\underline{0}}$ in pro- $\left(V \bar{e} c t_{F}\right)_{\underline{0}}$ holds as well, i.e., $S h_{\underline{0}}(X)=S h_{\underline{0}}(Y)$ in $S h_{\underline{0}}\left(V_{e c t}\right)$. Therefore, by Theorem 3.7, $\operatorname{dim} X=\operatorname{dim} Y$.

Corollary 4.3 (ii) shows that the quotient shape classifications of normed vectorial spaces might be strictly coarser than the isomorphism classification. In order to avoid general quotient linear morphisms (in some important cases) and to construct a desired example, we firstly need the next definition and lemma (see also Remark 3.6). We also need the following important consequence of the proof of Theorem 4.2.

Corollary 4.4. Let $X=(V,\|\cdot\|) \in O b\left(N V e c t_{F}\right)$ and let

$$
\boldsymbol{p}_{\kappa^{-}}=\left(p_{\lambda}\right): X \rightarrow \boldsymbol{X}_{\kappa^{-}}=\left(X_{\lambda}=\left(V_{\lambda},\|\cdot\|_{\lambda}\right), p_{\lambda \lambda^{\prime}}, \Lambda_{\kappa^{-}}\right)
$$

be an $\left(N V e c t_{F}\right)_{\kappa^{-}}$-expansion of $X$. Suppose that there exists a norm $\|\cdot\|^{\prime}$ on $V$ such that, for every $\lambda \in \Lambda_{\kappa^{-}}$, the quotient norm $\|\cdot\|_{\lambda}^{\prime}$ (on $V_{\lambda}$ ) is equivalent to $\|\cdot\|_{\lambda}$. If the identity function $1_{V}: X \rightarrow Y=\left(V,\|\cdot\|^{\prime}\right)$ is continuous, then

$$
\boldsymbol{q}_{\kappa^{-}}=\left(q_{\lambda}=p_{\lambda}\right): Y \rightarrow \boldsymbol{X}_{\kappa^{-}}
$$

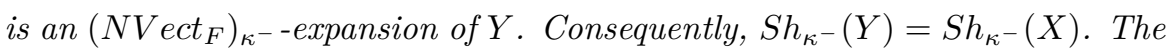
same holds in the $\kappa$-case.

Proof. By the proof of Theorem 4.2, for every $\lambda \in \Lambda_{\kappa^{-}}$, the quotient functions $p_{\lambda}$ and $q_{\lambda}$ coincide, and they are continuous and open. Since $\|\cdot\|_{\lambda}^{\prime}$ and $\|\cdot\|_{\lambda}$ are equivalent, $\lambda \in \Lambda_{\kappa^{-}}$, it follows that

$$
\boldsymbol{q}_{\kappa^{-}}=\left(q_{\lambda}=p_{\lambda}\right): Y=(V, \mathcal{S}) \rightarrow \boldsymbol{X}_{\kappa^{-}}
$$

is a morphism of pro- $\left(N V e c t_{F}\right)_{\kappa^{-}}$and that

$$
\left(Y_{\lambda}=\left(V_{\lambda},\|\cdot\|_{\lambda}^{\prime}\right), p_{\lambda \lambda^{\prime}}, \Lambda_{\kappa^{-}}\right) \cong\left(X_{\lambda}=\left(V_{\lambda},\|\cdot\|_{\lambda}\right), p_{\lambda \lambda^{\prime}}, \Lambda_{\kappa^{-}}\right)
$$

in $\operatorname{pro}^{-}\left(N V e c t_{F}\right)_{\kappa^{-}}$. Thus, since

$$
\boldsymbol{p}_{\kappa^{-}}=\left(p_{\lambda}\right): X \rightarrow \boldsymbol{X}_{\kappa^{-}}=\left(X_{\lambda}=\left(V_{\lambda},\|\cdot\|_{\lambda}\right), p_{\lambda \lambda^{\prime}}, \Lambda_{\kappa^{-}}\right)
$$

is an $\left(N V e c t_{F}\right)_{\kappa^{-}}$expansion of $X$ and $1_{V}: X \rightarrow Y$ is continuous, the factorization property for $\boldsymbol{q}_{\kappa^{-}}$follows by (E1) of $\boldsymbol{p}_{\kappa^{-}}$. Therefore, $\boldsymbol{q}_{\kappa^{-}}$is an $\left(N V e c t_{F}\right)_{\kappa^{-}}$expansion of $Y$ (having the same inverse system $\boldsymbol{X}_{\kappa^{-}}$). The proof of the $\kappa$-case works in the same manner. 
In order to obtain a non-trivial example, we firstly introduce an auxiliary notion.

DeFinition 4.5. Let $J$ be a set, let $V \unlhd F^{J}$ be a vectorial subspace of $F^{J}=\{x \mid x: J \rightarrow F\}$, and let $\|\cdot\|: V \rightarrow \mathbb{R}$ be a norm on $V$. The norm $\|\cdot\|$ is said to be monotone if the following condition is fulfilled:

$$
((\forall x, y \in V)(\forall j \in J)|x(j)| \leq|y(j)|) \Rightarrow\|x\| \leq\|y\| .
$$

Question. Is every norm on every $V \unlhd F^{J}$ monotone $(F \in\{\mathbb{Q}, \mathbb{R}, \mathbb{C}\})$ ?

Lemma 4.6. Let $J$ be a well ordered set. For every $j \in J$, denote $F^{j} \equiv$ $F^{[\min J, j]}$. Suppose that the vectorial space $F^{J}$ admits a norm $\|\cdot\|$ which is monotone. Then, for every $j \in J$, there exists a norm $\|\cdot\|_{j}$ on the vectorial space $F^{j}$ such that the projection

$$
p_{j}:\left(F^{J},\|\cdot\|\right) \rightarrow\left(F^{j},\|\cdot\|_{j}\right), \quad p_{j}(x)=x \mid[\min J, j],
$$

is an open continuous epimorphism. Furthermore, for every related pair $j \leq$ $j^{\prime}$ in $J$, the projection

$$
p_{j j^{\prime}}:\left(F^{j^{\prime}},\|\cdot\|_{j^{\prime}}\right) \rightarrow\left(F^{j},\|\cdot\|_{j}\right), \quad p_{j j^{\prime}}\left(x^{\prime}\right)=x^{\prime} \mid[\min J, j],
$$

is an open continuous epimorphism as well. The same holds true for the direct $\kappa$-sum $F_{\kappa^{-}}^{J} \unlhd F^{J}$ carrying a monotone norm $\|\cdot\|$, whenever $\aleph_{0} \leq \kappa \leq|J|$.

Proof. Given a $j \in J$, let the vectorial subspace $Z^{j} \equiv\left\{x \in F^{J}\right.$ $\left.x\left(j^{\prime}\right)=0, j^{\prime}>j\right\} \unlhd F^{J}$ carries the norm $\|\cdot\|_{j}^{\prime}$ that is the restriction of $\|\cdot\|$. Let $p_{j}^{\prime}:\left(F^{J},\|\cdot\|\right) \rightarrow\left(Z^{j},\|\cdot\|_{j}^{\prime}\right)$ be the projection. Then, obviously, $p_{j}^{\prime}$ is a linear epimorphism. Since, for every $x \in F^{J}$,

$$
p_{j}(x)\left(j^{\prime}\right)= \begin{cases}x\left(j^{\prime}\right), & j^{\prime} \leq j \\ 0, & j^{\prime}>j\end{cases}
$$

it follows that, for every $j^{\prime} \in J,\left|p_{j}^{\prime}(x)\left(j^{\prime}\right)\right| \leq\left|x\left(j^{\prime}\right)\right|$. Thus, for every $x \in F^{J}$, $\left\|p_{j}^{\prime}(x)\right\| \leq\|x\|\left(\right.$ in $\left.\left(F^{J},\|\cdot\|\right)\right)$ because $\|\cdot\|$ is monotone. Now, given any $\varepsilon>0$, it follows that

$$
\|x\|<\delta=\varepsilon \Rightarrow\left\|p_{j}^{\prime}(x)\right\|_{j}^{\prime}=\left\|p_{j}^{\prime}(x)\right\| \leq\|x\|<\varepsilon .
$$

This proves that $p_{j}^{\prime}$ is continuous at the null-vector $\theta$. Since $p_{j}^{\prime}$ is a linear function, the continuity of $p_{j}^{\prime}$ follows. Further, let an $r>0$ be given, and let $B(\theta, r)$ be the $r$-ball in $\left(F^{J},\|\cdot\|\right)$ at $\theta$. We are to prove that

$$
p_{j}^{\prime}[B(\theta, r)]=B_{j}(\theta, r),
$$

where $B_{j}(\theta, r)$ is the $r$-ball in $\left(Z^{j},\|\cdot\|_{j}^{\prime}\right)$ at $p_{j}^{\prime}(\theta)=\theta$. Let $x \in p_{j}^{\prime}[B(\theta, r)] \subseteq Z^{j}$. Then $x=p_{j}^{\prime}\left(x^{\prime}\right)$ for some $x^{\prime} \in B(\theta, r) \subseteq F^{J}$. Since

$$
\|x\|_{j}^{\prime}=\|x\|=\left\|p_{j}^{\prime}\left(x^{\prime}\right)\right\| \leq\left\|x^{\prime}\right\|<r,
$$


it follows that $x \in B j(\theta, r)$, and thus, $p_{j}^{\prime}[B(\theta, r)] \subseteq B_{j}(\theta, r)$. Conversely, if $x \in B_{j}(\theta, r) \subseteq Z^{j} \subseteq F^{J}$, then $\|x\|_{j}^{\prime}<r$ and $p_{j}^{\prime}(x)=x$. Hence,

$$
\|x\|=\|x\|_{j}^{\prime}<r
$$

and thus, $x \in B(\theta, r)$ and $x=p_{j}^{\prime}(x) \in p_{j}^{\prime}[B(\theta, r)]$. Therefore, $p_{j}^{\prime}$ is open at the null-vector $\theta$. Again, the linearity of $p_{j}^{\prime}$ implies that it is an open mapping. In a similar way one can prove that each $p_{j j^{\prime}}^{\prime}: Z_{j^{\prime}} \rightarrow Z_{j}, j \leq j^{\prime}$, is an open continuous epimorphism. Finally, it remains to observe that, for every $j \in J$, the vectorial space $F^{j}$ can be canonically algebraically identified with $Z^{j}$, as well as $p_{j}$ with $p_{j}^{\prime}$ and $p_{j j^{\prime}}$ with $p_{j j^{\prime}}^{\prime}$, whenever $j \leq j^{\prime}$. Therefore, the conclusion follows by putting $\|\cdot\|_{j}$ on $F^{j}$ to be the same as the restriction $\|\cdot\|_{j}^{\prime}$ of $\|\cdot\|$ to $Z^{j}$. In the case of the direct $\kappa$-sum $F_{\kappa^{-}}^{J} \unlhd F^{J}$, for a given $\kappa$ such that $\aleph_{0} \leq \kappa \leq|J|$ (see Remark 3.6), the proof works in the same manner.

EXAmPLE 4.7. For each $p \in \mathbb{R}, 1 \leq p<\infty$, and each ordered pair $a<b \in$ $\mathbb{R}$, let $C_{p}[a, b]$ denote the normed vectorial space $\left(C[a, b],\|\cdot\|_{p}\right)$, where $C[a, b]$ is the real $(F=\mathbb{R})$ vectorial space of all continuous functions $x:[a, b] \rightarrow \mathbb{R}$, and

$$
\|x\|_{p}=\left(\int_{a}^{b}|x(t)|^{p} d t\right)^{\frac{1}{p}} .
$$

Then no pair of $C_{p}[a, b], C_{p^{\prime}}[a, b], p \neq p^{\prime}$, is isomorphic in $N V e c t_{\mathbb{R}}$. We are to prove that all the normed spaces $C_{p}[a, b]$ have the same finite quotient shape. Firstly, by the well-known Jensen's inequality

$$
\varphi\left(\int_{a}^{b} f(t) d t\right) \leq \int_{a}^{b} \varphi(f(t)) d t
$$

( $\varphi$ - a convex function, $f(t) \geq 0$ ), the following inequality holds true:

$$
\left(\forall 1 \leq p \leq p^{\prime}<\infty\right)(\exists \alpha>0)(\forall x \in C[a, b])\|x\|_{p} \leq \alpha\|x\|_{p^{\prime}} .
$$

We shall prove it, for instance, in the technically simplest case, i.e., for $a=0$ and $b=1$. (Then $\alpha=1$.), Since $\frac{p^{\prime}}{p} \geq 1$, the function $\varphi:\{0\} \cup \mathbb{R}^{+} \rightarrow \mathbb{R}$, $\varphi(t)=t^{\frac{p^{\prime}}{p}}$, is convex. Thus,

$$
\begin{aligned}
\left(\|x\|_{p}\right)^{p^{\prime}} & =\left(\int_{0}^{1}|x(t)|^{p} d t\right)^{\frac{p^{\prime}}{p}}=\varphi\left(\int_{0}^{1}|x(t)|^{p} d t\right) \\
& \leq \int_{0}^{1} \varphi\left(|x(t)|^{p}\right) d t=\int_{0}^{1}\left(|x(t)|^{p}\right)^{\frac{p^{\prime}}{p}} d t=\int_{0}^{1}|x(t)|^{p^{\prime}} d t=\left(\|x\|_{p^{\prime}}\right)^{p^{\prime}}
\end{aligned}
$$

and hence, $\|x\|_{p} \leq\|x\|_{p^{\prime}}$. Consequently, the identity function $1_{C[a, b]}$ : $C[a, b] \rightarrow C[a, b]$, regarded as

$$
1_{p}^{p^{\prime}}: C_{p^{\prime}}[a, b] \rightarrow C_{p}[a, b]
$$


becomes continuous. Now the conclusion,

$$
\left(\forall 1 \leq p \leq p^{\prime}<\infty\right) S h_{\underline{0}}\left(C_{p}[a, b]\right)=S h_{\underline{0}}\left(C_{p^{\prime}}[a, b]\right),
$$

follows by Corollary 4.4 because all the norms on a finite-dimensional vectorial space are equivalent.

EXAMPLE 4.8. Let us consider, for all $1 \leq p \leq \infty$, the well known normed vectorial spaces $l_{p}$. Recall that $l_{p}=\left(\left\{x=\left.\left(x_{i}\right) \in F^{\mathbb{N}}\left|\Sigma_{i \in \mathbb{N}}\right| x_{i}\right|^{p}<\infty\right\},\|\cdot\|_{p}\right)$, $\|x\|_{p}=\left(\Sigma_{i \in \mathbb{N}}\left|x_{i}\right|^{p}\right)^{\frac{1}{p}}, 1 \leq p<\infty$, while $l_{\infty}=\left(\left\{x=\left(x_{i}\right) \in F^{\mathbb{N}} \mid x\right.\right.$ bounded $\left.\},\|\cdot\|_{\infty}\right),\|x\|_{\infty}=\sup \left\{\left|x_{i}\right| \mid i \in \mathbb{N}\right\}$. Of course, no pair $l_{p}, l_{p^{\prime}}, p \neq p^{\prime}$, is isomorphic in $N V e c t_{F}$. However, algebraically, for all $1 \leq p \leq p^{\prime} \leq \infty$, it holds $l_{1} \unlhd l_{p} \unlhd l_{p^{\prime}} \unlhd l_{\infty} \unlhd F^{\mathbb{N}}$ (in $V e c t_{F}$; coordinatewise operations). Furthermore, one readily sees that, for every $p, \operatorname{dim} l_{p}>\aleph_{0}$. Then, since $|F| \leq 2^{\aleph_{0}}$, Lemma 3.3 (ii) implies that, for every $p, \operatorname{dim} l_{p}=\operatorname{dim} F^{\mathbb{N}}=2^{\aleph_{0}}$. Therefore, for every $p, l_{p} \cong F^{\mathbb{N}}$ algebraically (in $V e c t_{F}$ ). For every related pair $p \leq p^{\prime}$, denote by $l_{p}\left(p^{\prime}\right) \unlhd l_{p^{\prime}}$ the corresponding normed vectorial subspace of $l_{p^{\prime}}$. Clearly, $l_{p}(p)=l_{p}$, while $l_{p}\left(p^{\prime}\right)$ is not isomorphic in $N V$ ect $F$ to $l_{p}$, whenever $p<p^{\prime}$. Notice now that

$$
\left(\forall 1 \leq p \leq p^{\prime} \leq \infty\right)\left(\forall x \in l_{p}\right)\|x\|_{p^{\prime}} \leq\|x\|_{p}
$$

holds true. Indeed, since $\frac{p^{\prime}}{p} \geq 1, p^{\prime}<\infty$, it follows that

$$
\left(\|x\|_{p^{\prime}}\right)^{p^{\prime}}=\Sigma_{i \in \mathbb{N}}\left|x_{i}\right|^{p^{\prime}}=\Sigma_{i \in \mathbb{N}}\left(\left|x_{i}\right|^{p}\right)^{\frac{p^{\prime}}{p}} \leq\left(\Sigma_{i \in \mathbb{N}}\left|x_{i}\right|^{p}\right)^{\frac{p^{\prime}}{p}}=\left(\|x\|_{p}\right)^{p^{\prime}},
$$

and thus, $\|x\|_{p^{\prime}} \leq\|x\|_{p}$. Further, if $p^{\prime}=\infty$, then

$$
\|x\|_{\infty}=\sup \left\{\left|x_{i}\right| \mid i \in \mathbb{N}\right\} \leq\left(\Sigma_{i \in \mathbb{N}}\left|x_{i}\right|^{p}\right)^{\frac{1}{p}}=\|x\|_{p} .
$$

This implies that, for all $p \leq p^{\prime}$, the inclusion $j_{p^{\prime}}^{p}: l_{p} \hookrightarrow l_{p^{\prime}}$ is continuous. Consequently, the identity function $1_{p}: l_{p} \rightarrow l_{p}$ becomes continuous regarded as

$$
1_{p}\left(p^{\prime}\right): l_{p} \rightarrow l_{p}\left(p^{\prime}\right) .
$$

Finally, recall that all norms on a finite-dimensional vectorial space are equivalent. Now, by applying Corollary 4.4, it follows that, for all $1 \leq p \leq p^{\prime} \leq \infty$,

$$
S h_{\underline{0}}\left(l_{p}\left(p^{\prime}\right)\right)=S h_{\underline{0}}\left(l_{p}\right) .
$$

REMARK 4.9. (a) We do not know whether the space $l_{p^{\prime}}$ and its subspace $l_{p}\left(p^{\prime}\right) \unlhd l_{p^{\prime}}$ have the same finite shape. (The affirmative answer would imply that all the normed vectorial spaces $l_{p}$ have the same finite shape.) This would be immediately followed by, for instance, $l_{p} \cong\left(F^{\mathbb{N}},\|\cdot\|_{p}^{\prime}\right)$ such that $\|\cdot\|_{p^{\prime}}^{\prime} \leq\|\cdot\|_{p}^{\prime}\left(\right.$ or $\left.\|\cdot\|_{p^{\prime}}^{\prime} \geq\|\cdot\|_{p}^{\prime}\right)$ whenever $p \leq p^{\prime}$. However, we do not know whether such norms $\|\cdot\|_{p}^{\prime}$ on $F^{\mathbb{N}}$ exist. Related to this, it seems that the subcategory of all normed vectorial spaces (over an $F$ ) admitting a topological 
(or, especially, Schauder) basis cannot bring any essential benefit, except some technical simplifications.

(b) Since the quotient space of a Banach space by a closed subspace is again a Banach space, there are the quotient shape theories for Banach spaces which yield non-trivial classifications as well. Further, the same holds for normed algebras, because they are preserved by quotients with respect to the closed both sides ideals.

Quite similarly to Example 4.8, the next example holds as well.

ExAmPLE 4.10. Let $F \in\{\mathbb{Q}, \mathbb{R}, \mathbb{C}\}$ and let $F_{0}^{\mathbb{N}} \unlhd F^{\mathbb{N}}$ be the direct sum of $F_{i} \equiv F$. Consider, for every $1 \leq p \leq \infty$, the well known norm $\|\cdot\|_{p}$, i.e., $\|x\|_{p}=\left(\Sigma_{i=1}^{n(x)}\left|x_{i}\right|^{p}\right)^{\frac{1}{p}}, p \neq \infty$, and $\|x\|_{\infty}=\max \left\{\left|x_{i}\right| \mid i \in \mathbb{N}\right\}$ on $F_{0}^{\mathbb{N}}$. Then all the normed vectorial spaces $\left(F_{0}^{\mathbb{N}},\|\cdot\|_{p}\right), 1 \leq p \leq \infty$, have the same finite shape, i.e., for all $1 \leq p, p^{\prime} \leq \infty$,

$$
S h_{\underline{0}}\left(F_{0}^{\mathbb{N}},\|\cdot\|_{p}\right)=S h_{\underline{0}}\left(F_{0}^{\mathbb{N}},\|\cdot\|_{p^{\prime}}\right) .
$$

4.2. The general case. Let $X=(V, \mathcal{T})$ be a topological vectorial space over $F$ and let $Z=(W, \mathcal{T} \mid W) \unlhd X$ be a vectorial and topological subspace of $X$. Let

$$
p: V \rightarrow V / W, \quad p(x) \equiv[x]=x+W,
$$

be the corresponding quotient in $V e c t_{F}$. Let $V / W$ carry the quotient topology $\mathcal{T}(p)$ with respect to $\mathcal{T}$ and the projection $p$. We are to prove that $(V / W, \mathcal{T}(p))$ is a topological vectorial space over $F$, which is called the quotient topological vectorial space of $X$ by $Z$, denoted by $X / Z$, and that linear epimorphism

$$
p: X \rightarrow X / Z, \quad p(x) \equiv[x]=x+Z,
$$

is continuous and open. In the case of a $T_{2}$-topological vectorial space $X$, a subspace $Z$ has to be closed. However, we do not know whether that necessary condition is also sufficient for a $T_{2}$-topology on $X / Z$. Thus, we shall hereby consider the quotient shapes in the most general case only.

LEMMA 4.11. For every topological vectorial space $X=(V, \mathcal{T})$ over $F$ and every topological vectorial subspace $Z=(W, \mathcal{T} \mid W) \unlhd X$, the quotient function $p: X \rightarrow X / Z$ is a linear continuous open epimorphism and $X / Z$ is a topological vectorial space over $F$.

Proof. Clearly, the quotient function $p: X \rightarrow X / Z$ is a linear epimorphism already on the algebraic level, i.e., as $p: V \rightarrow V / W$. Further, $p$ is continuous by definition. $(\mathcal{T}(p)$ is the largest topology on $V / W$ making $p$ to be continuous of $X$.). In order to prove that $p$ is an open mapping, let us denote, for every $A \subseteq X$,

$$
\widetilde{A}=\cup_{x \in A}[x]=\cup_{x \in A}(x+Z) \subseteq X .
$$


By Lemma 7.3 of [10] (see also [4], VI, 4.2(2)), $p$ is open if and only if, for every $U \subseteq X$ open, the set $\widetilde{U} \subseteq X$ is open. Since

$$
\begin{aligned}
\widetilde{U} & =\cup_{x \in U}(x+Z)=\{x+z \mid x \in U, z \in Z\} \\
& =\{z+x \mid z \in Z, x \in U\}=\cup_{z \in Z}(z+U),
\end{aligned}
$$

and since $z+U$ is homeomorphic (linearly isomorphic) to $U$, it is open as well. Therefore, every such $\widetilde{U}$, being a union of open sets, is an open set. It remains to prove that the operations on $X / Z$ (functions of $X / Z \times X / Z$ and $F \times X / Z$ to $X / Z)$

$$
\begin{aligned}
{[x]+z[y] } & =[x+y]=(x+y)+Z \text { and } \\
\alpha \cdot z[x] & =[\alpha x]=(\alpha x)+Z
\end{aligned}
$$

are continuous. In order to do it, let us observe that the diagrams

$$
\begin{array}{ccccccc}
X \times X & \stackrel{+}{*} & X & & F \times X & \rightarrow & X \\
\downarrow p \times p & & \downarrow p \\
X / Z \times X / Z & \stackrel{+}{\rightarrow} & X / Z & \text { and } & \downarrow 1_{F} \times p & & \downarrow p \\
& & F \times X / Z & \stackrel{\text { ‘ }}{\rightarrow} & X / Z
\end{array}
$$

commute (in $V e c t_{F}$ ). Furthermore, since $X / Z$ carries the quotient topology (with respect to $\mathcal{T}$ and $p$ ) and $p$ is an open mapping, the direct product topology on $X / Z \times X / Z$ and the quotient topology with respect to $p \times p$ coincide. So the left diagram shows that $+_{Z}$ is continuous. Similarly, since $p$ and $1_{F}$ are open mappings, the direct product topology on $F \times X / Z$ and the quotient topology with respect to $1_{F} \times p$ coincide. Thus, the right diagram shows that $\cdot Z$ is continuous.

THEOREM 4.12. For each infinite cardinal $\kappa \geq \aleph_{0}$, the categories $\left(\text { TVect }_{F}\right)_{\kappa^{-}}$and $\left(T V e c t_{F}\right)_{\kappa}$ are pro-reflective subcategories of TVect . $_{\text {. }}$ Moreover, if $X=(V, \mathcal{T}) \in O b\left(\right.$ TVect $\left._{F}\right)$ and

$$
\begin{gathered}
\boldsymbol{p}_{\kappa^{-}}=\left(p_{\lambda}\right): V \rightarrow \boldsymbol{V}_{\kappa^{-}}=\left(V_{\lambda}, p_{\lambda \lambda^{\prime}}, \Lambda_{\kappa^{-}}\right) \\
\left(\boldsymbol{p}_{\kappa}=\left(p_{\lambda}\right): V \rightarrow \boldsymbol{V}_{\kappa}=\left(V_{\lambda}, p_{\lambda \lambda^{\prime}}, \Lambda_{\kappa}\right)\right)
\end{gathered}
$$

is a $\left(\text { Vect }_{F}\right)_{\kappa^{-}-\text {expansion }}\left(\left(\text { Vect }_{F}\right)_{\kappa^{-}}\right.$expansion $)$of $V$, then

$$
\begin{gathered}
\boldsymbol{p}_{\kappa^{-}}=\left(p_{\lambda}\right): X \rightarrow \boldsymbol{X}_{\kappa^{-}}=\left(X_{\lambda}, p_{\lambda \lambda^{\prime}}, \Lambda_{\kappa^{-}}\right) \\
\left(\boldsymbol{p}_{\kappa}=\left(p_{\lambda}\right): X \rightarrow \boldsymbol{X}_{\kappa}=\left(X_{\lambda}, p_{\lambda \lambda^{\prime}}, \Lambda_{\kappa}\right)\right)
\end{gathered}
$$

is a $\left(\text { TVect }{ }_{F}\right)_{\kappa^{-}}$-expansion $\left(\left(T V e c t_{F}\right)_{\kappa^{-}}\right.$expansion) of $X$, whenever, for every $\lambda \in \Lambda_{\kappa^{-}}\left(\lambda \in \Lambda_{\kappa}\right)$, the topology $\mathcal{T}_{\lambda}$ of $X_{\lambda}=\left(V_{\lambda}, \mathcal{T}_{\lambda}\right)$ is the quotient topology (with respect to $\mathcal{T}$ and $p_{\lambda}$ ). 
Proof. By Theorem 3.1, $\left(V e c t_{F}\right)_{\kappa^{-}}$and $\left(V e c t_{F}\right)_{\kappa}$ are pro-reflective subcategories of $V e c t_{F}$. Let $X \in O b\left(T V e c t_{F}\right)$ and let

$$
\begin{aligned}
\boldsymbol{p}_{\kappa^{-}} & =\left(p_{\lambda}\right): X \rightarrow \boldsymbol{X}_{\kappa^{-}}=\left(X_{\lambda}, p_{\lambda \lambda^{\prime}}, \Lambda_{\kappa^{-}}\right), \quad \text { and } \\
\boldsymbol{p}_{\kappa} & =\left(p_{\lambda}\right): X \rightarrow \boldsymbol{X}_{\kappa}=\left(X_{\lambda}, p_{\lambda \lambda^{\prime}}, \Lambda_{\kappa}\right)
\end{aligned}
$$

be the algebraic (quotient, see Theorem 3.1) $\left(\text { Vect }_{F}\right)_{\kappa^{-}}$-expansion and $\left(V e c t_{F}\right)_{\kappa}$-expansion of the (underlying) vectorial space $X$ respectively. (We have written $X, X_{\lambda}$ instead of $V, V_{\lambda}$ because the word "algebraic" and notation $V e c t_{F}$ cause no ambiguity!). Now, by assuming the quotient topology on each $X_{\lambda}$ (with respect to $p_{\lambda}$ of $X$ ), the linear epimorphisms $p_{\lambda \lambda^{\prime}}$ are continuous too. Further, Lemma 4.11 implies that $\boldsymbol{p}_{\kappa^{-}}$and $\boldsymbol{p}_{\kappa}$ are morphisms of pro- $\left(T V e c t_{F}\right)_{\kappa^{-}}$and pro- $\left(T V e c t_{F}\right)_{\kappa}$ respectively. We need verify the expansion factorization property (E1), i.e., we have to prove the continuity of $f_{\lambda}$ in the algebraic factorization $f_{\lambda} p_{\lambda}=f$ (see the proof of Theorem 3.1), whenever $f$ is continuous. Let $Y \in O b\left(T V e c t_{F}\right)_{\kappa^{-}}$and let $f: X \rightarrow Y$ be a continuous linear function. Then there exists an algebraic factorization $f_{\lambda} p_{\lambda}=f$. We have to prove that $f_{\lambda}: X_{\lambda} \rightarrow Y$ is continuous. Let $V \subseteq Y$ be any open set. Then $f^{-1}[V] \subseteq X$ is open. Since $X_{\lambda}$ carries the quotient topology with respect to $p_{\lambda}$ and $\mathcal{T}$, the set $f_{\lambda}^{-1}[V] \subseteq X_{\lambda}$ is open if and only if, $p_{\lambda}^{-1}\left[f_{\lambda}^{-1}[V]\right] \subseteq X$ is open. Since $p_{\lambda}^{-1}\left[f_{\lambda}^{-1}[V]\right]=\left(f_{\lambda} p_{\lambda}\right)^{-1}[V]=f^{-1}[V]$, the conclusion follows. The proof of the $\kappa$-case is the same.

Theorem 4.12 supports the following corollaries (compare Corollaries 4.4 and 4.3).

Corollary 4.13. Let $X=(V, \mathcal{T}) \in O b\left(T V e c t_{F}\right)$ and let

$$
\boldsymbol{p}_{\kappa^{-}}=\left(p_{\lambda}\right): X \rightarrow \boldsymbol{X}_{\kappa^{-}}=\left(X_{\lambda}=\left(V_{\lambda}, \mathcal{T}_{\lambda}\right), p_{\lambda \lambda^{\prime}}, \Lambda_{\kappa^{-}}\right)
$$

be a $\left(\text { TVect }{ }_{F}\right)_{\kappa^{-}}$expansion of $X$. Suppose that there exists a topology $\mathcal{S}$ on $V$ such that, for every $\lambda \in \Lambda_{\kappa^{-}}$, the quotient topology $\mathcal{S}_{\lambda}=\mathcal{T}_{\lambda}$ (on $V_{\lambda}$ ). Then

$$
\boldsymbol{q}_{\kappa^{-}}=\left(q_{\lambda}=p_{\lambda}\right): Y=(V, \mathcal{S}) \rightarrow \boldsymbol{X}_{\kappa^{-}}
$$

is a $\left(\text { TVect }{ }_{F}\right)_{\kappa^{-}}$expansion of $Y$, and thus,

$$
S h_{\left(T V e c t_{F}\right) \kappa^{-}}(Y)=S h_{\left(T V e c t_{F}\right) \kappa^{-}}(X) .
$$

The same holds in the $\kappa$-case.

Proof. Observe that

$$
\boldsymbol{p}_{\kappa^{-}}=\boldsymbol{q}_{\kappa^{\cdot}}=\left(p_{\lambda}=q_{\lambda}\right): V \rightarrow\left(V_{\lambda}, p_{\lambda \lambda^{\prime}}, \Lambda_{\kappa}\right)
$$

is the same (algebraic) $\left(V e c t_{F}\right)_{\kappa^{-}}$-expansion of both $X$ and $Y$, (i.e., an expansion of $V)$. Further, the quotient topologies $\mathcal{T}_{\lambda}$ and $\mathcal{S}_{\lambda}, \lambda \in \Lambda_{\kappa^{-}}$, coincide. Thus the conclusion immediately follows by Theorem 4.12.

Corollary 4.14. Let $X, Y \in O b\left(\right.$ TVect $\left._{F}\right)$ such that $S h_{\kappa^{-}}(X)=$ $S h_{\kappa^{-}}(Y)$, for some $\kappa \leq \operatorname{dim} X$. Then, 
(i) $\operatorname{dim} X<\infty \Rightarrow Y \cong X\left(\right.$ in $_{\text {TVect }}$ );

(ii) $\operatorname{dim} X=\infty \Rightarrow \operatorname{dim} Y=\operatorname{dim} X$.

Proof. It suffices to prove the statements in the case $\kappa=\aleph_{0}$.

(i) Since $X$ is finite-dimensional, $\left\lfloor 1_{X}\right\rfloor: X \rightarrow\lfloor X\rfloor$ is a rudimentary finitedimensional (and a rudimentary $\kappa^{-}$- and $\kappa$-, for every $\kappa \geq \aleph_{0}$ ) expansion of $X$. Consequently, $S h_{0}(X)=S h_{0}(Y)$ implies that $Y$ admits a rudimentary finite-dimensional expansion $\lfloor q\rfloor: Y \rightarrow\lfloor X\rfloor$. Therefore, $q: Y \rightarrow X$ is an isomorphism of $T V$ ect $_{F}$.

(ii) Let $\boldsymbol{p}_{\underline{0}}=\left(p_{\lambda}\right): X \rightarrow \boldsymbol{X}_{\underline{0}}=\left(X_{\lambda}, p_{\lambda \lambda^{\prime}}, \Lambda_{\underline{0}}\right)$ and $\boldsymbol{q}_{\underline{0}}=\left(q_{\mu}\right): Y \rightarrow \boldsymbol{Y}_{\underline{0}}=$ $\left(Y_{\mu}, q_{\mu \mu^{\prime}}, M_{\underline{0}}\right)$ be appropriate expansions of $X$ and $Y$ respectively. Then, by forgetting the topologies, they are the corresponding algebraic expansions as well. Thus, the final conclusion follows by Theorem 3.7.

Corollaries 4.13 and 4.14 (ii) show that the quotient shape classifications of topological vectorial spaces should be strictly coarser than the isomorphism (linear homeomorphism) classification. Here is the simplest general example.

EXAMPLE 4.15. Let $F$ be a field carrying the discrete topology, and let $X=\left(F^{\mathbb{N}}, \mathcal{T}\right)$ be the countable direct product space of $F_{i}=F$ (i.e., $\mathcal{T}$ is the product topology that is not discrete). Then $\left(F^{\mathbb{N}}, \mathcal{T}\right)$ is a topological vectorial space because, for every $n \in \mathbb{N}$,

$$
\begin{aligned}
& p_{n} \circ+=+_{n} \circ\left(p_{n} \times p_{n}\right) \quad \text { and } \\
& p_{n} \circ \cdot=\cdot_{n} \circ\left(1_{F} \times p_{n}\right)
\end{aligned}
$$

$\left(p_{n}: F^{\mathbb{N}} \rightarrow F^{n}\right.$ - the projection), which implies that + and $\cdot$ on $F^{\mathbb{N}}$ are continuous with respect to $\mathcal{T}$ (compare the proof of Lemma 4.11). Let

$$
\boldsymbol{p}_{\underline{0}}=\left(p_{\lambda}\right): X \rightarrow \boldsymbol{X}_{\underline{0}}=\left(X_{\lambda}, p_{\lambda \lambda^{\prime}}, \Lambda_{\underline{0}}\right)
$$

be the $\left(T V e c t_{F}\right)_{0^{-}}$expansion of $X$ obtained via the algebraic expansion of $F^{\mathbb{N}}$. Then, for every $\lambda \in \Lambda_{\underline{0}}, X_{\lambda} \cong F^{n(\lambda)}$ carries the discrete topology. Further, let $Y=\left(F^{\mathbb{N}}, \mathcal{S}\right)$, where $\mathcal{S}$ is the discrete topology. Then

$$
\boldsymbol{q}_{\underline{0}}=\left(q_{\lambda}=p_{\lambda}\right): Y \rightarrow \boldsymbol{Y}_{\underline{0}}=\boldsymbol{X}_{\underline{0}}
$$

is a $\left(T V e c t_{F}\right)_{\underline{0}^{-}}$expansion of $Y$. By Corollary 4.13, $X$ and $Y$ have the same finite quotient shape,

$$
S h_{\underline{0}}(X)=S h_{\underline{0}}(Y),
$$

while $X$ and $Y$ are not isomorphic objects of $T V$ ect $_{F}$, because they are not mutually homeomorphic topological spaces. Observe that the fields $\mathbb{Z}_{p}, p \in \mathbb{N}$, $p \geq 2$ a prime number, give rise of such concrete examples.

Since every normed vectorial space $X=(V,\|\cdot\|)$ is a topological (metric) vectorial space, denoted by $X^{\prime}=\left(V, \mathcal{T}_{d}\right)\left(d\left(x, x^{\prime}\right)=\left\|x-x^{\prime}\right\|\right)$, one may consider its quotient shapes in both categories - NVect ${ }_{F}$ and $T V e c t_{F}$. Firstly, observe that $X \cong Y=\left(W,\|\cdot\|^{\prime}\right)$ in $N V$ ect $t_{F}$ is equivalent to $X^{\prime} \cong Y^{\prime}$ in $T V e c t_{F} t$. 
Namely (for the converse implication in the case $W=V$ ), if $\mathcal{T}_{d}=\mathcal{T}_{d^{\prime}}$ on $V$, then the metrics $d$ and $d^{\prime}$ (obtained by norms $\|\cdot\|$ and $\|\cdot\|^{\prime}$ respectively) are (uniformly) equivalent, implying that the norms $\|\cdot\|$ and $\|\cdot\|^{\prime}$ on $V$ are equivalent. The general case, i.e., $W \cong V$, reduces to $W=V$ (see the appropriate part of proof of Corollary 4.16 below).

The final result clarifies that relationship.

Corollary 4.16. Let $X=(V,\|\cdot\|)$ and $Y=\left(W,\|\cdot\|^{\prime}\right)$ be normed vectorial spaces and let $X^{\prime}=\left(V, \mathcal{T}_{d}\right)$ and $Y^{\prime}=\left(W, \mathcal{T}_{d^{\prime}}\right)$ be the corresponding topological vectorial spaces. Then, for every $\kappa \geq \aleph_{0}$, the following equivalence holds:

$$
S h_{\kappa^{-}}(X)=S h_{\kappa^{-}}(Y) \Leftrightarrow S h_{\kappa^{-}}\left(X^{\prime}\right)=S h_{\kappa^{-}}\left(Y^{\prime}\right) .
$$

Proof. First of all, notice that, in general,

$$
X \cong Y\left(\text { in } N V \text { ect }_{F} t\right) \Leftrightarrow X^{\prime} \cong Y^{\prime}(\text { in } T V \text { ect } F t)
$$

holds true. Indeed, $\operatorname{dim} X=\operatorname{dim} X^{\prime}$ holds by definition, while $\operatorname{dim} X=\operatorname{dim} Y$ and $\operatorname{dim} X^{\prime}=\operatorname{dim} Y^{\prime}$ hold by Corollaries 4.3 (ii) and 4.14 (ii) respectively. Now, in the finite-dimensional case the equivalence holds trivially. In the infinite-dimensional case, by Lemma $3.3\left(\operatorname{dim} F \geq 2^{\aleph_{0}}\right)$ and Remark 3.6, there exists a $\kappa_{1} \geq \aleph_{0}$ such that both $V$ and $W$ are isomorphic to the direct $\kappa_{1}$-sum $F_{\kappa_{1}^{-}}^{J} \unlhd \sqcap_{j \in J} F_{j} \equiv F^{j}$, where $|J|=\kappa_{1}$. Let $f: F_{\kappa_{1}^{-}}^{J} \rightarrow V$ be an isomorphism. Put

$$
\left(\forall u \in F_{\kappa_{1}^{-}}^{J}\right)\|u\|_{1}=\|f(u)\| .
$$

Then $\|\cdot\|_{1}$ is a norm on $F_{\kappa_{1}^{-}}^{J}$ and $f:\left(F_{\kappa_{1}^{-}}^{J},\|\cdot\|_{1}\right) \rightarrow(V,\|\cdot\|)=X$ is an isometrical isomorphism of $N V$ ect $_{F}$, In the same way, there exists an isometrical isomorphism $f^{\prime}:\left(F_{\kappa_{1}^{-}}^{J},\|\cdot\|_{1}^{\prime}\right) \rightarrow(W,\|\cdot\|)=Y$. Hence, $X^{\prime} \cong\left(F_{\kappa_{1}^{-}}^{J}, \mathcal{T}_{d_{1}}\right)$ and $Y^{\prime} \cong\left(F_{\kappa_{1}^{-}}^{J}, \mathcal{T}_{d_{1}^{\prime}}\right)$ in $T V e c t_{F}$, where the metric topologies $\mathcal{T}_{d_{1}}$ and $\mathcal{T}_{d_{1}^{\prime}}$ are induced by the norms $\|\cdot\|_{1}$ and $\|\cdot\|_{1}^{\prime}$ on $F_{\kappa_{1}^{-}}^{J}$ respectively. Consequently, the stated equivalence reduces to

$$
S h_{\kappa^{-}}\left(F_{\kappa_{1}^{-}}^{J},\|\cdot\|_{1}\right)=S h_{\kappa^{-}}\left(F_{\kappa_{1}^{-}}^{J},\|\cdot\|_{1}^{\prime}\right) \Leftrightarrow S h_{\kappa^{-}}\left(F_{\kappa_{1}^{-}}^{J}, \mathcal{T}_{d_{1}}\right)=S h_{\kappa^{-}}\left(F_{\kappa_{1}^{-}}^{J}, \mathcal{T}_{d_{1}^{\prime}}\right), \kappa_{1} \geq \kappa .
$$

Now, the necessity follows by Theorem 4.12 and Corollary 4.13 (or as a special case of the more general one). For the converse implication, since $\mathcal{T}_{d_{1} j}=\mathcal{T}_{d_{1}^{\prime} j}$, for all appropriate pairs of terms of the corresponding expansions, the metrics $d_{1 j}$ and $d_{1 j}^{\prime}$ are (uniformly) equivalent, and thus the norms $\|\cdot\|_{1 j}$ and $\|\cdot\|_{1 j}^{\prime}$ on all appropriate pairs of terms are equivalent. The conclusion follows.

REMARK 4.17. Notice that one can relate the obtained fact to the standard shape theory (of topological spaces). Namely, for every topological space $X, \boldsymbol{p}=\left(p_{n}\right): X^{\mathbb{N}} \rightarrow\left(X^{n}, p_{n n^{\prime}}, \mathbb{N}\right)$, is the inverse limit (in Top). If, especially, $X$ is a compact polyhedron or a compact $A N R$, then $\boldsymbol{p}$ yields

$$
H \boldsymbol{p}=\left(\left[p_{n}\right]\right): X^{\mathbb{N}} \rightarrow\left(X^{n},\left[p_{n n^{\prime}}\right], \mathbb{N}\right)
$$


(in pro-HTop), that is a HPol-expansion of $X^{\mathbb{N}}$ ([9], I. 6, Theorems 1 and 2). Consequently, if, for instance, $X=F=\mathbb{Z}_{p}$ - discrete, where $p \geq 2$ is a prime number, an interesting result (example) relating the standard shape theory of $S h(T o p)$ and the quotient one of $S h_{\underline{0}}\left(T V e c t_{\mathbb{Z}_{p}}\right)$ occurs.

\section{REFERENCES}

[1] K. Borsuk, Concerning homotopy properties of compacta, Fund. Math. 62 (1968), $223-254$.

[2] K. Borsuk, Theory of Shape, Monografie Matematyczne 59, Polish Scientific Publishers, Warszawa, 1975.

[3] J.-M. Cordier and T. Porter, Shape Theory: Categorical Methods of Approximation, Ellis Horwood Ltd., Chichester, 1989. (Dover edition, 2008.)

[4] J. Dugundji, Topology, Allyn and Bacon, Inc., Boston, 1978.

[5] J. Dydak and J. Segal, Shape theory: An introduction, Lecture Notes in Math. 688, Springer-Verlag, Berlin, 1978.

[6] H. Herlich and G. E. Strecker, Category Theory: An Introduction, Allyn and Bacon Inc., Boston, 1973.

[7] S. Kurepa, Funkcionalna analiza: elementi teorije operatora, Školska knjiga, Zagreb, 1990.

[8] N. Koceić Bilan and N. Uglešić, The coarse shape, Glas. Mat. Ser. III 42(62) (2007), $145-187$.

[9] S. Mardešić and J. Segal, Shape Theory, North-Holland, Amsterdam, 1982.

[10] N. Uglešić, The shapes in a concrete category, Glas. Mat. Ser. III 51(71) (2016), 255-306.

[11] N. Uglešić and B. Červar, The concept of a weak shape type, Int. J. Pure Appl. Math. 39 (2007), 363-428.

\section{O kvocientnim oblicima vektorskih prostora}

\section{Nikica Uglešić}

SAŽETAK. U radu se razmatraju kvocientni oblici vektorskih prostora - algebarski i topoložki (posebice, za normirane prostore). U algebarskomu slučaju se sve oblikovne razredbe svode na izomorfnost. U topoložkomu (i, posebice, normiranomu) slučaju, međutim, oblikovne razredbe su bitno grublje od izomorfnosti.

Veli Råt, Dugi Otok, Hrvatska

E-mail: nuglesic@unizd.hr

Received: 14.9.2016.

Revised: 24.2.2017. 